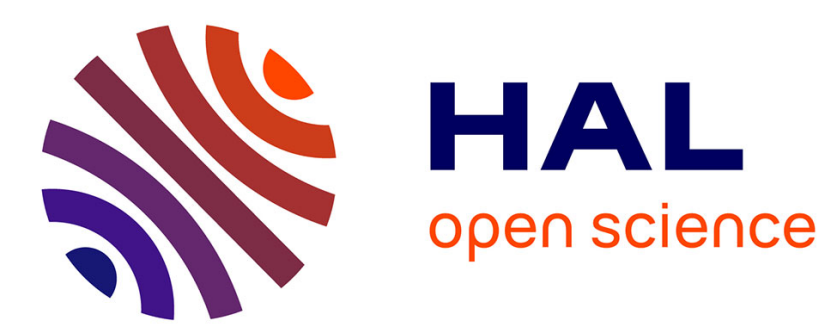

\title{
Shakedown of elastic-perfectly plastic materials with temperature-dependent elastic moduli
}

\author{
Michaël Peigney
}

\section{To cite this version:}

Michaël Peigney. Shakedown of elastic-perfectly plastic materials with temperature-dependent elastic moduli. Journal of the Mechanics and Physics of Solids, 2014, 71, pp.112-131. 10.1016/j.jmps.2014.06.008 . hal-01055869

\section{HAL Id: hal-01055869 \\ https://hal-enpc.archives-ouvertes.fr/hal-01055869}

Submitted on 13 Aug 2014

HAL is a multi-disciplinary open access archive for the deposit and dissemination of scientific research documents, whether they are published or not. The documents may come from teaching and research institutions in France or abroad, or from public or private research centers.
L'archive ouverte pluridisciplinaire HAL, est destinée au dépôt et à la diffusion de documents scientifiques de niveau recherche, publiés ou non, émanant des établissements d'enseignement et de recherche français ou étrangers, des laboratoires publics ou privés. 


\title{
Shakedown of elastic-perfectly plastic materials with temperature-dependent elastic moduli
}

\author{
Michaël Peigney \\ Université Paris-Est, Laboratoire Navier (Ecole des Ponts ParisTech, IFSTTAR, CNRS), F-77455 \\ Marne-la-Vallée Cedex 2, France, \\ michael.peigney@polytechnique.org
}

\begin{abstract}
For elastic-perfectly plastic materials with constant properties, the well-known Melan's theorem gives a sufficient condition for shakedown to occur, independently on the initial state. It has been conjectured that Melan's theorem could be extended to temperature-dependent (or time-dependent) elastic moduli, but no theoretical result is available. This paper aims at providing results in that direction, with a special emphasis on time-periodic variations. If Melan's condition is satisfied, we show that shakedown indeed occurs provided the time fluctuations of the elastic moduli satisfy a certain condition (which in particular is fulfilled if the time fluctuations are not too large). We provide a counterexample which shows that setting such a constraint on the elastic moduli is necessary to reach path-independent theorems as proposed. A simple mechanical system is studied as an illustrative example.
\end{abstract}

Key words: elastic-plastic materials, temperature-dependent material properties, Melan's theorem, shakedown

\section{Introduction}

For elastic-perfectly plastic structures under prescribed loading histories, the celebrated Melan's theorem (Melan, 1936; Symonds, 1951; Koiter, 1960) gives a sufficient condition for the evolution to become elastic in the large-time limit. That situation, classically referred to as shakedown, is associated with the intuitive idea that the plastic strain tends to a limit as time tends to infinity. The Melan's theorem has the distinctive property of being path-independent, i.e. independent on the initial state of the structure. For a parametrized loading history, Melan's theorem gives bounds on the domain of load parameters for which shakedown occurs. Regarding fatigue design, shakedown corresponds to the most beneficial regime of high-cycle fatigue, as opposed to the regime of low-cycle fatigue which typically occurs if the plastic strain does not converge towards a stabilized value (DangVan and Papadopoulos, 1999).

The contribution of Koiter (1960) gave rise to a lot of subsequent research, mainly along two different lines. A first line of research is concerned with extensions of the original theorem to various nonlinear behaviors, such as hardening plasticity (Pham, 2008; Nguyen, 2003), non standard plasticity (Corigliano et al., 1995; Bodovillé and De Saxcé, 2001), contact with friction (Ahn et al., 2008), phase-transformation (Peigney, 2010). A second (and complementing) line of research is concerned with the development of numerical methods for efficiently determining the shakedown 
domain in the space of load parameters (Zarka et al., 1988; Maitournam et al., 2002; Carvelli et al., 1999; Peigney and Stolz, 2001, 2003; Simon and Weichert, 2012; Spiliopoulos and Panagiotou, 2012). We refer to Weichert and Ponter (2014) for more historical details on the development of shakedown theory.

In this paper, we aim at extending Melan's theorem to situations in which the elastic moduli are fluctuating in time. Such time fluctuations may result from significant variations of the temperature. In a lot of practical situations, structural elements are indeed submitted to thermomechanical loading histories in which variations of the temperature are large enough for the temperature dependence of the material not to be negligible. The case of the temperature-dependent yield limits has been considered by Borino (2000). In contrast, the case of temperature-dependent elastic moduli remains a long standing issue, even though the practical importance of that problem has been recognized early (König, 1969).

In order to underline the fundamental difficulties raised by temperature-dependent moduli, we observe that, for elastic-plastic materials with a constant elastic tensor, the distance between two solutions is monotonically decreasing with time (more details are given in Sec. 3). That property plays a crucial role in proving shakedown theorems, both in the original framework considered by Koiter as well as in most of the extensions mentioned earlier. That property does not to hold anymore for temperature-dependent elastic moduli, which indicates that temperature-dependent moduli fundamentally change the mathematical nature of the evolution problem.

Using incremental analysis, the asymptotic behavior of some specific mechanical systems has been studied by Halphen and di Domizio (2005); Hasbroucq et al. (2010, 2012). The obtained results led to some conjectures about shakedown theorems for time-dependent elastic moduli, but not theoretical result is available. This paper aims at providing some results in that direction, and is organized as follows. In Sec. 2 we establish the differential inclusion that governs the evolution of the stress field, Eq. (15), and discuss the relation between the equation obtained and the sweeping process introduced by Moreau (1977). The main goal of the paper is to study the asymptotic behavior of the stress field, defined as a solution of Eq. (15) for some given initial condition. The well-known case of time-independent elastic moduli is briefly discussed in Sec. 3. We present the shakedown condition originally introduced by Koiter (1960) as well as two variations of that condition. Although those three conditions are interrelated, they are not strictly equivalent, as we shall explain. The most conclusive results are obtained when the space of residual stress fields is of finite dimension. In that case, under any of the three conditions introduced in Sec. 3, the stress field can be proved to converge towards an elastic response, independently on the initial state. The question is whether the same conclusions can be extended to time-dependent elastic moduli. We show in Sec. 4 that the general answer is no: considering time-periodic variations of the elastic moduli, we provide an explicit counterexample for which there exists an inelastic limit cycle, even though the conditions discussed in Sec. 3 are satisfied. For that example, the solutions of (15) are either attracted to an inelastic limit cycle or to the set of elastic solutions, depending on the initial state. This ruins any hope that the conditions of Sec. 3 are sufficient for shakedown to occur in a path-independent fashion. Positive results are given in Sec. 5-6: we show that shakedown theorems can still be obtained for time-dependent elastic moduli, but their formulation differs significantly from the classical Melan's theorem. A key point in the analysis is a general bound on the variations of the elastic energy, Eq. (50), that we establish in Sec. 5. That result is used in Sec. 6 to prove some shakedown theorems when the elastic moduli are time-periodic. Those theorems are applied in Sec. 7 to a simple mechanical system. Numerical results of incremental analysis are also provided to illustrate the ideas discussed throughout the paper. 


\section{Evolution equation for the stress field}

Consider an elastic-perfectly plastic material occupying a domain $\Omega$ in the reference configuration. Under the assumption of infinitesimal strains, the strain tensor $\epsilon$ is derived from the displacement $\boldsymbol{u}$ by

$$
\boldsymbol{\epsilon}=\frac{1}{2}\left(\nabla \boldsymbol{u}+\nabla^{T} \boldsymbol{u}\right)
$$

The strain $\boldsymbol{\epsilon}$, stress $\boldsymbol{\sigma}$ and plastic strain $\boldsymbol{\epsilon}^{p}$ at position $\boldsymbol{x}$ and time $t$ satisfy the constitutive equations

$$
\begin{gathered}
\boldsymbol{\epsilon}(\boldsymbol{x}, t)=\boldsymbol{L}(\boldsymbol{x}, t): \boldsymbol{\sigma}+\boldsymbol{\epsilon}^{\theta}(\boldsymbol{x}, t)+\boldsymbol{\epsilon}^{p}(\boldsymbol{x}, t), \\
\dot{\boldsymbol{\epsilon}}^{p}(\boldsymbol{x}, t) \in \partial I_{\mathcal{C}(\boldsymbol{x}, t)}(\boldsymbol{\sigma}(\boldsymbol{x}, t)) .
\end{gathered}
$$

In (1), $\boldsymbol{L}$ is the (symmetric positive definite) elastic moduli tensor and $\boldsymbol{\epsilon}^{\theta}$ is the thermal strain tensor. The double product : in (1) denotes contraction with respect to the last two indexes, i.e. $(\boldsymbol{L}: \boldsymbol{\sigma})_{i j}=\sum_{k, l} \boldsymbol{L}_{i j k l} \boldsymbol{\sigma}_{l k}$. In (2), $\mathcal{C}$ is the elasticity domain of the material, assumed to be closed and convex. The space and time dependence of $\boldsymbol{L}, \boldsymbol{\epsilon}^{\theta}$ and $\mathcal{C}$ may result from imposed variations of the temperature. For instance, the elastic properties of most materials are known to depend on the temperature $\theta$. Such a relation can be written in the form $\boldsymbol{L}=\boldsymbol{L}(\theta)$. For imposed variations $\theta(\boldsymbol{x}, t)$ of the temperature, the elastic moduli tensor $\boldsymbol{L}(\theta(\boldsymbol{x}, t))$ can be regarded as a function of space and time.

The function $I_{\mathcal{C}(\boldsymbol{x}, t)}$ in (2) is the indicator function of $\mathcal{C}(\boldsymbol{x}, t)$ (equal to 0 in $\mathcal{C}(\boldsymbol{x}, t)$, infinite otherwise) and the operator $\partial$ denotes the subgradient (Brézis, 1972; Rockafellar, 1997). The general definition of the subgradient is recalled for latter reference: for a function $\phi$ defined on a Hilbert space $H, \partial \phi$ is the multi-valued mapping defined by

$$
\partial \phi(\boldsymbol{\tau})=\left\{\boldsymbol{a}:\left(\boldsymbol{\tau}^{\prime}-\boldsymbol{\tau}, \boldsymbol{a}\right)_{H} \leq \phi\left(\boldsymbol{\tau}^{\prime}\right)-\phi(\boldsymbol{\tau}) \forall \boldsymbol{\tau}^{\prime} \in H\right\}
$$

where $(., .)_{H}$ is the scalar product on $H$. In the case $\phi=I_{K}$ where $K$ is a closed convex set, the definition (3) specializes as

$$
\partial I_{K}(\boldsymbol{\tau})= \begin{cases}\emptyset & \text { if } \boldsymbol{\tau} \notin K \\ \left\{\boldsymbol{a}:\left(\boldsymbol{\tau}^{\prime}-\boldsymbol{\tau}, \boldsymbol{a}\right)_{H} \leq 0 \forall \boldsymbol{\tau}^{\prime} \in K\right\} & \text { if } \boldsymbol{\tau} \in K\end{cases}
$$

In particular, Eq. (2) implicitly enforces that

$$
\boldsymbol{\sigma}(\boldsymbol{x}, t) \in \mathcal{C}(\boldsymbol{x}, t) .
$$

From (4) we obtain the inequality

$$
\dot{\boldsymbol{\epsilon}}^{p}:\left(\boldsymbol{\sigma}^{\prime}-\boldsymbol{\sigma}\right) \leq 0 \text { for all } \boldsymbol{\sigma}^{\prime} \in \mathcal{C}(\boldsymbol{x}, t)
$$

which corresponds to the principle of maximum plastic dissipation (Hill, 1950).

Body forces $\boldsymbol{f}(\boldsymbol{x}, t)$ are imposed in the domain $\Omega$ and given tractions $\boldsymbol{t}(\boldsymbol{x}, t)$ are prescribed on a part $\partial \Omega_{t}$ of the boundary $\partial \Omega$. Prescribed displacements $\boldsymbol{v}(\boldsymbol{x}, t)$ are imposed on $\partial \Omega_{u}=\partial \Omega-\partial \Omega_{t}$. Assuming quasi-static evolutions, the stress field $\boldsymbol{\sigma}$ satisfies the equilibrium equations

$$
\operatorname{div} \boldsymbol{\sigma}+\boldsymbol{f}=0 \text { in } \Omega, \boldsymbol{\sigma} \cdot \boldsymbol{n}=\boldsymbol{t} \text { on } \partial \Omega_{t} .
$$


We now derive the equation governing the evolution of the stress field. Let $\mathcal{E}$ be the space of stress fields with square-integrable components. It is known that $\mathcal{E}$ is a Hilbert space for the scalar product

$$
\left\langle\boldsymbol{\sigma}, \boldsymbol{\sigma}^{\prime}\right\rangle=\int_{\Omega} \boldsymbol{\sigma}(\boldsymbol{x}): \boldsymbol{\sigma}^{\prime}(\boldsymbol{x}) d \omega .
$$

The associated norm is denoted by \|\| , i.e. $\|\boldsymbol{\sigma}\|=\sqrt{\langle\boldsymbol{\sigma}, \boldsymbol{\sigma}\rangle}$.

Let $\left(\boldsymbol{u}^{E}, \boldsymbol{\sigma}^{E}\right)$ be the fictitious elastic response of the system, that is the solution of

$$
\begin{gathered}
\boldsymbol{\epsilon}^{E}=\boldsymbol{L}(\boldsymbol{x}, t): \boldsymbol{\sigma}^{E}+\boldsymbol{\epsilon}^{\theta}(\boldsymbol{x}, t), \\
\boldsymbol{\epsilon}^{E}=\frac{1}{2}\left(\nabla \boldsymbol{u}^{E}+\nabla^{T} \boldsymbol{u}^{E}\right), \\
\operatorname{div} \boldsymbol{\sigma}^{E}+\boldsymbol{f}=0 \text { in } \Omega, \boldsymbol{\sigma}^{E} \cdot \boldsymbol{n}=\boldsymbol{t} \text { on } \partial \Omega_{t}, \\
\boldsymbol{u}^{E}=\boldsymbol{v} \text { on } \partial \Omega_{u} .
\end{gathered}
$$

The stress field $\boldsymbol{\sigma}$ can be decomposed as $\boldsymbol{\sigma}=\boldsymbol{\sigma}^{E}+\boldsymbol{\rho}$ where $\boldsymbol{\rho}$ is the residual stress field and belongs to the vectorial space

$$
\mathcal{H}=\left\{\boldsymbol{\rho} \in \mathcal{E}: \operatorname{div} \boldsymbol{\rho}=0 \text { in } \Omega, \boldsymbol{\rho} \cdot \boldsymbol{n}=0 \text { on } \partial \Omega_{t}\right\} .
$$

Let $\mathcal{K}_{0}(t)$ and $\mathcal{K}(t)$ be respectively the convex subsets of $\mathcal{E}$ and $\mathcal{H}$ defined as

$$
\mathcal{K}_{0}(t)=\{\boldsymbol{\sigma} \in \mathcal{E}: \boldsymbol{\sigma}(\boldsymbol{x}, t) \in \mathcal{C}(\boldsymbol{x}, t) \forall \boldsymbol{x} \in \Omega\}
$$

and

$$
\mathcal{K}(t)=\left\{\boldsymbol{\rho} \in \mathcal{H}: \boldsymbol{\rho}+\boldsymbol{\sigma}^{E}(t) \in \mathcal{K}_{0}(t)\right\} .
$$

The set $\mathcal{K}_{0}(t)$ is the set of plastically admissible stress fields. The set $\mathcal{K}(t)$ is the set of self-stress fields such that $\boldsymbol{\rho}+\boldsymbol{\sigma}^{E}$ is plastically admissible. Under suitable regularity assumptions on $(\boldsymbol{f}, \boldsymbol{t}, \boldsymbol{v})$, it can be proved that the sets $\mathcal{H}$ and $\mathcal{K}(t)$ are closed in $\mathcal{E}$ (Lanchon, 1974).

For an arbitrary $\boldsymbol{\rho}^{\prime} \in \mathcal{K}(t)$, it follows from (1) that

$$
\int_{\Omega}\left(\boldsymbol{\rho}^{\prime}-\boldsymbol{\rho}\right): \frac{d\left(\boldsymbol{\epsilon}-\boldsymbol{\epsilon}^{E}\right)}{d t} d \omega=\int_{\Omega}\left(\boldsymbol{\rho}^{\prime}-\boldsymbol{\rho}\right): \frac{d}{d t}(\boldsymbol{L}(\boldsymbol{x}, t): \boldsymbol{\rho}) d \omega+\int_{\Omega}\left(\boldsymbol{\rho}^{\prime}-\boldsymbol{\rho}\right): \dot{\boldsymbol{\epsilon}}^{p} d \omega .
$$

Since $\boldsymbol{\rho}+\boldsymbol{\sigma}^{E} \in \mathcal{K}_{0}(t)$ and $\boldsymbol{\rho}^{\prime}+\boldsymbol{\sigma}^{E} \in \mathcal{K}_{0}(t)$, Eq. (5) gives

$$
\left(\boldsymbol{\rho}^{\prime}-\boldsymbol{\rho}\right): \dot{\boldsymbol{\epsilon}}^{p}=\left(\boldsymbol{\rho}^{\prime}+\boldsymbol{\sigma}^{E}-\boldsymbol{\rho}-\boldsymbol{\sigma}^{E}\right): \dot{\boldsymbol{\epsilon}}^{p} \leq 0 .
$$

Therefore

$$
\int_{\Omega}\left(\boldsymbol{\rho}^{\prime}-\boldsymbol{\rho}\right): \frac{d\left(\boldsymbol{\epsilon}-\boldsymbol{\epsilon}^{E}\right)}{d t} d \omega \leq \int_{\Omega}\left(\boldsymbol{\rho}^{\prime}-\boldsymbol{\rho}\right): \frac{d}{d t}(\boldsymbol{L}(\boldsymbol{x}, t): \boldsymbol{\rho}) d \omega .
$$

Using (7-8) together with the principle of virtual power shows that

$$
\int_{\Omega}\left(\boldsymbol{\rho}^{\prime}-\boldsymbol{\rho}\right): \frac{d\left(\boldsymbol{\epsilon}-\boldsymbol{\epsilon}^{E}\right)}{d t} d \omega=-\int_{\Omega} \operatorname{div}\left(\boldsymbol{\rho}^{\prime}-\boldsymbol{\rho}\right) \cdot \frac{d\left(\boldsymbol{u}-\boldsymbol{u}^{E}\right)}{d t}+\int_{\partial \Omega} \frac{d\left(\boldsymbol{u}-\boldsymbol{u}^{E}\right)}{d t} \cdot\left(\boldsymbol{\rho}^{\prime}-\boldsymbol{\rho}\right) \cdot \boldsymbol{n} d \Sigma
$$

Therefore (9) becomes

$$
-\int_{\Omega}\left(\boldsymbol{\rho}^{\prime}-\boldsymbol{\rho}\right): \frac{d}{d t}(\boldsymbol{L}(\boldsymbol{x}, t): \boldsymbol{\rho}) d \omega \leq 0 \forall \boldsymbol{\rho}^{\prime} \in \mathcal{K}(t) .
$$


From the general definition (4), Eq. (10) can be rewritten as

$$
-\frac{d}{d t}(\boldsymbol{L}(\boldsymbol{x}, t): \boldsymbol{\rho}) \in \partial I_{\mathcal{K}_{(t)}}(\boldsymbol{\rho})
$$

where $\partial I_{\mathcal{K}_{(t)}}$ is the subgradient of $I_{\mathcal{K}_{(t)}}$ for the scalar product $\langle.,$.$\rangle . The uniqueness of the stress$ rate $\dot{\boldsymbol{\rho}}$ has been proved by Halphen (2005) using variational principles for the differential inclusion (11).

Eq. (11) can be rewritten in a reduced form that proves to be useful for our analysis. Let $\boldsymbol{\pi}$ be the orthogonal projector on $\mathcal{H}$ for the scalar product $\langle.,$.$\rangle . For any \boldsymbol{\sigma} \in \mathcal{E}, \boldsymbol{\pi} \boldsymbol{\sigma}$ is characterized by

$$
\begin{aligned}
\boldsymbol{\pi} \boldsymbol{\sigma} & \in \mathcal{H}, \\
\left\langle\boldsymbol{\sigma}-\boldsymbol{\pi} \boldsymbol{\sigma}, \boldsymbol{\rho}^{\prime}\right\rangle & =0 \quad \forall \boldsymbol{\rho}^{\prime} \in \mathcal{H} .
\end{aligned}
$$

Eq. (10) gives $-\left\langle\boldsymbol{\rho}^{\prime}-\boldsymbol{\rho}, \boldsymbol{\pi} \frac{d}{d t}(\boldsymbol{L}(\boldsymbol{x}, t): \boldsymbol{\rho})\right\rangle \leq 0$ for all $\boldsymbol{\rho}^{\prime} \in \mathcal{K}(t)$. Therefore

$$
-\boldsymbol{\pi} \frac{d}{d t}(\boldsymbol{L}(\boldsymbol{x}, t): \boldsymbol{\rho}) \in \partial I_{\mathcal{K}_{(t)}}(\boldsymbol{\rho}) .
$$

Consider the linear operator $\mathcal{L}(t)$ defined on $\mathcal{H}$ by the relation

$$
\mathcal{L}(t) \boldsymbol{\rho}=\boldsymbol{\pi}(\boldsymbol{L}(\boldsymbol{x}, t): \boldsymbol{\rho}) .
$$

The distinctive properties of the operator $\mathcal{L}(t)$ are

$$
\begin{gathered}
\mathcal{L}(t) \boldsymbol{\rho} \in \mathcal{H}, \\
\left\langle\boldsymbol{\rho}^{\prime}, \mathcal{L}(t) \boldsymbol{\rho}\right\rangle=\int_{\Omega} \boldsymbol{\rho}^{\prime}(\boldsymbol{x}): \boldsymbol{L}(\boldsymbol{x}, t): \boldsymbol{\rho}(\boldsymbol{x}) d \omega, \forall \boldsymbol{\rho}, \boldsymbol{\rho}^{\prime} \in \mathcal{H} .
\end{gathered}
$$

Since the elastic moduli tensor $\boldsymbol{L}(\boldsymbol{x}, t)$ is symmetric positive definite, the relations (13) show that $\mathcal{L}(t)$ is self-adjoint and positive definite. It can also be verified from (13) that

$$
\boldsymbol{\pi} \frac{d}{d t}(\boldsymbol{L}(\boldsymbol{x}, t): \boldsymbol{\rho})=\frac{d}{d t}(\mathcal{L}(t) \boldsymbol{\rho})
$$

so that Eq. (12) can be rewritten as

$$
-\frac{d}{d t}(\mathcal{L}(t) \boldsymbol{\rho}) \in \partial I_{\mathcal{K}_{(t)}}(\boldsymbol{\rho}) .
$$

To better explain the relations between Eqs. (15) and (11), we first observe that for any $\boldsymbol{\rho} \in \mathcal{K}(t), \boldsymbol{\eta} \in \partial I_{\mathcal{K}_{(t)}}(\boldsymbol{\rho})$ and $\boldsymbol{\eta}^{\prime} \in \mathcal{H}^{\perp}$, we have $\boldsymbol{\eta}+\boldsymbol{\eta}^{\prime} \in I_{\mathcal{K}_{(t)}}(\boldsymbol{\rho})$. This is a direct consequence of the definition (4) and of the inclusion $\mathcal{K}(t) \subset \mathcal{H}$. Therefore

$$
\partial I_{\mathcal{K}(t)}(\boldsymbol{\rho})=\mathcal{H} \cap \partial I_{\mathcal{K}_{(t)}}(\boldsymbol{\rho})+\mathcal{H}^{\perp}
$$

Using that last identity, projecting (11) on $\mathcal{H}$ and $\mathcal{H}^{\perp}$ respectively gives

$$
-\boldsymbol{\pi} \frac{d}{d t}(\boldsymbol{L}(\boldsymbol{x}, t): \boldsymbol{\rho}) \in \mathcal{H} \cap \partial I_{\mathcal{K}_{(t)}}(\boldsymbol{\rho}),
$$




$$
-\boldsymbol{\pi}^{\perp} \frac{d}{d t}(\boldsymbol{L}(\boldsymbol{x}, t): \boldsymbol{\rho}) \in \mathcal{H}^{\perp}
$$

where $\boldsymbol{\pi}^{\perp}=\boldsymbol{I}-\boldsymbol{\pi}$ is the projector on $\mathcal{H}^{\perp}$ and $\boldsymbol{I}$ is the identity. The relation (14) shows that Eqs. (15) and (16) are equivalent. Since $\boldsymbol{\pi}^{\perp}$ takes values in $\mathcal{H}^{\perp}$, Eq. (17) is automatically satisfied and does not set any restriction on $\boldsymbol{\rho}$ or on its time derivative. Therefore, all the information in the evolution equation (11) is actually contained in its projection (15) on $\mathcal{H}$. In the following we primarily use the reduced Eq. (15), which has the advantage of taking values in $\mathcal{H}$ whereas Eq.(11) takes values in the bigger space $\mathcal{E}$.

When the elastic moduli do not depend on time, the distinction between (11) and (15) can be avoided. Denoting by $\boldsymbol{L}_{0}(\boldsymbol{x})$ the time-independent value of $\boldsymbol{L}(\boldsymbol{x}, t)$ at point $\boldsymbol{x}$, Eq. (10) indeed becomes

$$
-\int_{\Omega}\left(\boldsymbol{\rho}^{\prime}-\boldsymbol{\rho}\right): \boldsymbol{L}_{0}(\boldsymbol{x}): \frac{d \boldsymbol{\rho}}{d t} d \omega \leq 0 \quad \forall \boldsymbol{\rho}^{\prime} \in \mathcal{K}(t) .
$$

Introducing the scalar product $\left\langle\boldsymbol{\sigma}, \boldsymbol{\sigma}^{\prime}\right\rangle_{L_{0}}=\int_{\Omega} \boldsymbol{\sigma}(\boldsymbol{x}): \boldsymbol{L}_{0}: \boldsymbol{\sigma}^{\prime}(\boldsymbol{x}) d \omega$, we obtain

$$
-\frac{d \boldsymbol{\rho}}{d t} \in \partial_{L_{0}} I_{\mathcal{K}(t)}(\boldsymbol{\rho})
$$

where $\partial_{L_{0}}$ denotes the subgradient for the scalar product $\langle., .\rangle_{L_{0}}$. In (18), the elastic moduli do not appear explicitly and the left-hand side takes values in $\mathcal{H}$, without any need of an extra projection step.

Eq. (18) is the form commonly used in theoretical studies of elastic-plastic media with timeindependent elastic moduli (Moreau, 1977; Halphen, 1976; Baillon and Haraux, 1977; Wesfreid, 1981). The generic form of (18) is known as a 'sweeping process' and has been extensively studied by Moreau (1977). The name 'sweeping process' refers to a geometric interpretation of Eq. (18): if $\boldsymbol{\rho}(t)$ (viewed as a moving point in $\mathcal{H}$ ) is strictly inside $\mathcal{K}(t)$ then the point $\boldsymbol{\rho}(t)$ stays at rest. Whenever $\boldsymbol{\rho}(t)$ reaches the boundary of $\mathcal{K}(t)$, the set $\mathcal{K}(t)$ 'drags' the point $\boldsymbol{\rho}$ along its inward normal cone, in such a way that $\boldsymbol{\rho}(t)$ remains within $\mathcal{K}(t)$. Initially motivated by elastic-plastic systems, the sweeping process (18) also plays an important role in unilateral contact problems (see e.g. Ballard (2010) for some recent developments).

Solutions of the sweeping process (18) enjoy some remarkable properties. In particular, for two arbitrary solutions $\boldsymbol{\rho}$ and $\boldsymbol{\rho}^{\prime}$, we have

$$
\begin{aligned}
\frac{1}{2} \frac{d}{d t} \int_{\Omega}\left(\boldsymbol{\rho}-\boldsymbol{\rho}^{\prime}\right): \boldsymbol{L}_{0}:\left(\boldsymbol{\rho}-\boldsymbol{\rho}^{\prime}\right) d \omega & =\left\langle\frac{d}{d t}\left(\boldsymbol{\rho}-\boldsymbol{\rho}^{\prime}\right), \boldsymbol{\rho}-\boldsymbol{\rho}^{\prime}\right\rangle_{L_{0}} \\
& =-\left\langle\partial_{L_{0}} I_{\mathcal{K}_{(t)}}(\boldsymbol{\rho})-\partial_{L_{0}} I_{\mathcal{K}_{(t)}}\left(\boldsymbol{\rho}^{\prime}\right), \boldsymbol{\rho}-\boldsymbol{\rho}^{\prime}\right\rangle_{L_{0}} \\
& \leq 0 .
\end{aligned}
$$

The distance between two solutions of (18) (as measured by the energy norm) is thus decreasing with time. That property plays a crucial role in studying the asymptotic behaviour of (18) as $t \rightarrow+\infty$ (Halphen, 1976; Baillon and Haraux, 1977; Wesfreid, 1981). A similar property does not generally hold for the differential inclusion (15). This is actually the main source of the difficulties that are raised by the time dependence of the elastic moduli.

In the following, we investigate the asymptotic behaviour of solutions to (15). A special role is played by elastic solutions, defined as follows: a solution $\boldsymbol{\rho}(t)$ to (15) is said to be elastic (on the time interval $[\tau,+\infty[)$ if

$$
\frac{d}{d t}(\mathcal{L}(t) \boldsymbol{\rho}(t))=0 \quad \forall t \geq \tau .
$$


As stated in the introduction, shakedown corresponds to the intuitive idea that the plastic strain tends to a limit as $t \rightarrow+\infty$. For time-dependent elastic moduli, this property translates to the convergence of the residual stress field towards an elastic solution. The residual stress field $\boldsymbol{\rho}(t)$ is indeed characterized by the relation

$$
\left\langle\boldsymbol{\rho}^{\prime}, \mathcal{L}(t) \boldsymbol{\rho}(t)\right\rangle=-\left\langle\boldsymbol{\rho}^{\prime}, \boldsymbol{\epsilon}^{p}(t)\right\rangle \quad \forall \boldsymbol{\rho}^{\prime} \in \mathcal{H}
$$

Formally, if $\boldsymbol{\epsilon}^{p}(t)$ tends to a time-independent limit $\boldsymbol{\epsilon}_{\infty}^{p}$, then $\boldsymbol{\rho}(t)$ varies asymptotically as $-\mathcal{L}(t)^{-1} \boldsymbol{\epsilon}_{\infty}^{p}$, i.e converges towards an elastic solution as defined in (20).

We are primarily interested in studying conditions in which all the solutions of (15) converge towards some elastic solution, immaterial of the initial state $\boldsymbol{\rho}(0)$. The situation is well understood in the case of time-independent moduli, as explained in the next section.

\section{Asymptotic behavior in the case of time-independent elastic moduli}

The elastic moduli $\boldsymbol{L}(\boldsymbol{x}, t)$ are assumed in this section to be time-independent, in which case elastic solutions (on $[\tau,+\infty[$ ) are stationary (on $[\tau,+\infty[$ ). A necessary condition for such a stationary solution to exist is

$$
\bigcap_{t \geq \tau} \mathcal{K}(t) \neq \emptyset
$$

for some $\tau$. In order to obtain global results on the asymptotic behavior, the condition (21) is not sufficient and needs to be complemented with additional requirements. Several choices are possible in this regard. Koiter (1960) originally introduced the following

Condition 1. There exists an elastic solution $\boldsymbol{\rho}^{*}(t)$ (on a time interval $[\tau,+\infty[$ ) and $m>1$ such that $\boldsymbol{\rho}^{*}(t)+m \boldsymbol{\sigma}^{E}(t) \in \mathcal{K}_{0}(t)$ for all $t \geq \tau$.

Under Condition 1, the dissipated energy can be proved to remain bounded in time, independently on the initial state (Koiter, 1960). For finite dimensional systems, the boundedness of the dissipation rightly implies the convergence of both the plastic strain and the residual stress towards time-independent limits (see e.g. Nguyen (2003)). If $\mathcal{H}$ is of infinite dimension, additional assumptions on $\mathcal{K}(t)$ are needed to reach similar conclusions (Débordes et al., 1976; Nguyen, 2003).

Let $B\left(\boldsymbol{\rho}^{*}, r\right)$ denote the ball of center $\boldsymbol{\rho}^{*}$ and radius $r$ in $\mathcal{E}$, i.e. $B\left(\boldsymbol{\rho}^{*}, r\right)=\left\{\boldsymbol{\rho} \in \mathcal{E}:\left\|\boldsymbol{\rho}-\boldsymbol{\rho}^{*}\right\| \leq r\right\}$. Instead of Condition 1 , consider the (closely related) following

Condition 2. There exists an elastic solution $\boldsymbol{\rho}^{*}(t)$ (on a time interval $[\tau,+\infty[$ ) and $r>0$ such that $B\left(\rho^{*}(t), r\right) \subset \mathcal{K}(t)$ for all $t \geq \tau$.

Under Condition 2, all the solutions of (15) can be proved to converge towards some timeindependent state. Consider indeed a solution $\rho$ of (15) and introduce the positive function

$$
f_{0}(t)=\frac{1}{2}\left\langle\boldsymbol{\rho}(t)-\boldsymbol{\rho}^{*}, \mathcal{L}_{0}\left(\boldsymbol{\rho}(t)-\boldsymbol{\rho}^{*}\right)\right\rangle
$$

where $\boldsymbol{\rho}^{*}$ satisfies Condition 2 and $\mathcal{L}_{0}$ denotes the time-independent value of $\mathcal{L}(t)$. Note from $(19)$ that $f_{0}$ is decreasing with time and therefore can be interpreted as a Lyapunov function for the evolution problem (15). 
By (15) there exists $\boldsymbol{\eta}(t) \in \partial I_{\mathcal{K}_{(t)}}(\boldsymbol{\rho}(t))$ such that

$$
-\mathcal{L}_{0} \frac{d \boldsymbol{\rho}(t)}{d t}=\boldsymbol{\eta}(t)
$$

We have

$$
\dot{f}_{0}(t)=-\left\langle\boldsymbol{\rho}(t)-\boldsymbol{\rho}^{*}, \boldsymbol{\eta}(t)\right\rangle .
$$

Integrating between $t=\tau$ and $t=T$ gives

$$
\int_{\tau}^{T}\left\langle\boldsymbol{\rho}(t)-\boldsymbol{\rho}^{*}, \boldsymbol{\eta}(t)\right\rangle d t=f_{0}(\tau)-f_{0}(T) \leq f_{0}(\tau)
$$

Observe by Condition 2 that $\boldsymbol{\rho}^{*}+r \boldsymbol{\eta} /\|\boldsymbol{\eta}\|$ is in $\mathcal{K}(t)$. Therefore, we have using (5)

$$
0 \leq\left\langle\boldsymbol{\eta}(t), \boldsymbol{\rho}(t)-\left(\boldsymbol{\rho}^{*}+r \frac{\boldsymbol{\eta}}{\|\boldsymbol{\eta}\|}\right)\right\rangle=\left\langle\boldsymbol{\eta}(t), \boldsymbol{\rho}(t)-\boldsymbol{\rho}^{*}\right\rangle-r\|\boldsymbol{\eta}(t)\|
$$

i.e.

$$
r\|\boldsymbol{\eta}(t)\| \leq\left\langle\boldsymbol{\rho}(t)-\boldsymbol{\rho}^{*}, \boldsymbol{\eta}(t)\right\rangle .
$$

Combining (23) and (24) yields

$$
\int_{\tau}^{T}\|\boldsymbol{\eta}(t)\| d t \leq \frac{f_{0}(\tau)}{r} .
$$

Therefore, $\int_{0}^{T}\|\boldsymbol{\eta}(t)\| d t$ converges towards a limit as $T \longrightarrow+\infty$. Since $\mathcal{E}$ is a Hilbert space, it follows that $\int_{0}^{T} \boldsymbol{\eta}(t) d t$ also converges towards a limit as $T \longrightarrow+\infty$ (see e.g. Rudin (1987) or Theorem 97 in Schwartz (1967)). Use of (22) finally shows that $\boldsymbol{\rho}(t)$ converges towards the time-independent limit $\boldsymbol{\rho}(0)-\mathcal{L}_{0}^{-1} \int_{0}^{\infty} \boldsymbol{\eta}(t) d t$.

It can be observed that the above reasoning holds if Condition 2 is replaced by the following Condition, which is slightly less restrictive:

Condition 3. There exists an elastic solution $\boldsymbol{\rho}^{*}(t)$ (on a time interval $[\tau,+\infty[$ ) and $r>0$ such that $\left(\mathcal{H} \cap B\left(\boldsymbol{\rho}^{*}(t), r\right)\right) \subset \mathcal{K}(t)$ for all $t \geq \tau$.

Since the operator $\mathcal{L}_{0}$ takes values in $\mathcal{H}$, Eq. $(22)$ indeed shows that $\boldsymbol{\eta}(t) \in \mathcal{H}$. Therefore, the condition $\left(\mathcal{H} \cap B\left(\boldsymbol{\rho}^{*}(t), r\right)\right) \subset \mathcal{K}(t)$ is sufficient to obtain (24) and the subsequent conclusions.

The three Conditions 1-3 are interrelated, and we now detail the relations between them. Condition 2 clearly always implies Condition 3. If the dimension of $\mathcal{H}$ is finite, it can readily be seen that Conditions 1 and 2 are equivalent. We thus have the following:

$$
\text { Condition } 1 \Longleftrightarrow \text { Condition } 2 \Longrightarrow \text { Condition } 3
$$

When $\mathcal{H}$ is of infinite dimension, Condition 2 implies Condition 1 but the converse is not necessarily true. Therefore we have the following relations:

$$
\text { Condition } 1 \Longleftarrow \text { Condition } 2 \Longrightarrow \text { Condition } 3
$$

When the elastic moduli are time-independent, any of the Conditions 1-3 allows some results on the asymptotic behaviour to be obtained. In particular, if $\mathcal{H}$ is of finite dimension, any of the Conditions 1-3 ensures that the residual stress field converges towards an elastic solution. The question is whether the same conclusions can be extended to time-dependent elastic moduli. In the next section, we provide a counterexample showing that none of the Conditions 1-3 is sufficient for shakedown to occur when the elastic moduli depend on time. 


\section{A counterexample}

We consider an example of the evolution equation (15) in a case where $\mathcal{E}=\mathcal{H}$ is of dimension 2. All the matrix representations in the following are relative to an orthonormal basis of $\mathcal{H}$. The coordinates in that basis are denoted by $x_{1}$ and $x_{2}$. The convex set $\mathcal{K}_{0}(t)$ is taken as $\mathcal{K}_{0}(t)=$ $\boldsymbol{R}(\omega t) \mathcal{D}_{0}$ where $\omega$ is a non-negative parameter, $\mathcal{D}_{0}$ is the ellipse with equation

$$
x_{1}^{2}+4 x_{2}^{2} \leq 1
$$

and

$$
\boldsymbol{R}(\Theta)=\left(\begin{array}{cc}
\cos \Theta & -\sin \Theta \\
\sin \Theta & \cos \Theta
\end{array}\right)
$$

is the rotation of angle $\Theta$. The fictitious elastic response $\boldsymbol{\sigma}^{E}(t)$ is taken as

$$
\boldsymbol{\sigma}^{E}(t)=\frac{\sqrt{3}}{2}\left(\begin{array}{c}
\cos \omega t \\
\sin \omega t
\end{array}\right)
$$

so that the set $\mathcal{K}(t)$ is equal to $\boldsymbol{R}(\omega t) \mathcal{D}$ where $\mathcal{D}$ is the ellipse defined by

$$
\left(x_{1}-\frac{\sqrt{3}}{2}\right)^{2}+4 x_{2}^{2} \leq 1 .
$$

For $\boldsymbol{\rho} \in \partial \mathcal{K}(t)$, we have

$$
\partial I_{\mathcal{K}(t)}(\boldsymbol{\rho})=\{\lambda \boldsymbol{n}(\boldsymbol{\rho}, \mathcal{K}(t)): \lambda \geq 0\}
$$

where $\boldsymbol{n}(\boldsymbol{\rho}, \mathcal{K}(t))$ is the outward normal to $\mathcal{K}(t)$ at point $\boldsymbol{\rho}$ (Fig. 1). We note that the ball $B\left(0,1-\frac{\sqrt{3}}{2}\right)$ is included in $\mathcal{D}$. Therefore, Condition 2 is satisfied by $\boldsymbol{\rho}^{*}=0$ and $r=1-\frac{\sqrt{3}}{2}$. As a consequence of the property (25), Conditions 1 and 3 are also satisfied.

The symmetric positive definite operator $\mathcal{L}(t)$ is taken as the $2 \pi$-periodic function defined as

$$
\mathcal{L}(t)=\boldsymbol{R}(\omega t) \boldsymbol{S}_{0} \boldsymbol{R}(-\omega t)
$$

with

$$
\boldsymbol{S}_{0}=\frac{1}{4}\left(\begin{array}{cc}
3 \sqrt{3} & 1 \\
1 & \sqrt{3}
\end{array}\right)
$$

Let $\boldsymbol{\rho}_{0}$ be the point on $\partial \mathcal{D}$ with coordinates $(\sqrt{3} / 2,-1 / 2)$. It can easily be verified that

$$
\boldsymbol{S}_{0} \boldsymbol{\rho}_{0}=\boldsymbol{R}\left(\frac{\pi}{2}\right) \boldsymbol{n}\left(\boldsymbol{\rho}_{0}, \mathcal{D}\right) .
$$

That last property implies that

$$
\hat{\boldsymbol{\rho}}(t)=\boldsymbol{R}(\omega t) \boldsymbol{\rho}_{0}=\left(\begin{array}{c}
\cos \left(\omega t-\frac{\pi}{6}\right) \\
\sin \left(\omega t-\frac{\pi}{6}\right)
\end{array}\right)
$$

is a solution of (15). We have indeed

$$
\begin{aligned}
-\frac{d}{d t}(\mathcal{L}(t) \hat{\boldsymbol{\rho}}(t)) & =-\frac{d}{d t}\left(\boldsymbol{R}(\omega t) \boldsymbol{S}_{0} \boldsymbol{\rho}_{0}\right)=-\omega \boldsymbol{R}\left(\omega t+\frac{\pi}{2}\right) \boldsymbol{S}_{0} \boldsymbol{\rho}_{0} \\
& =\omega \boldsymbol{R}(\omega t) \boldsymbol{n}\left(\boldsymbol{\rho}_{0}, \mathcal{D}\right)=\omega \boldsymbol{n}(\hat{\boldsymbol{\rho}}(t), \mathcal{K}(t)) \in \partial I_{\mathcal{K}_{(t)}}(\hat{\boldsymbol{\rho}}(t)) .
\end{aligned}
$$




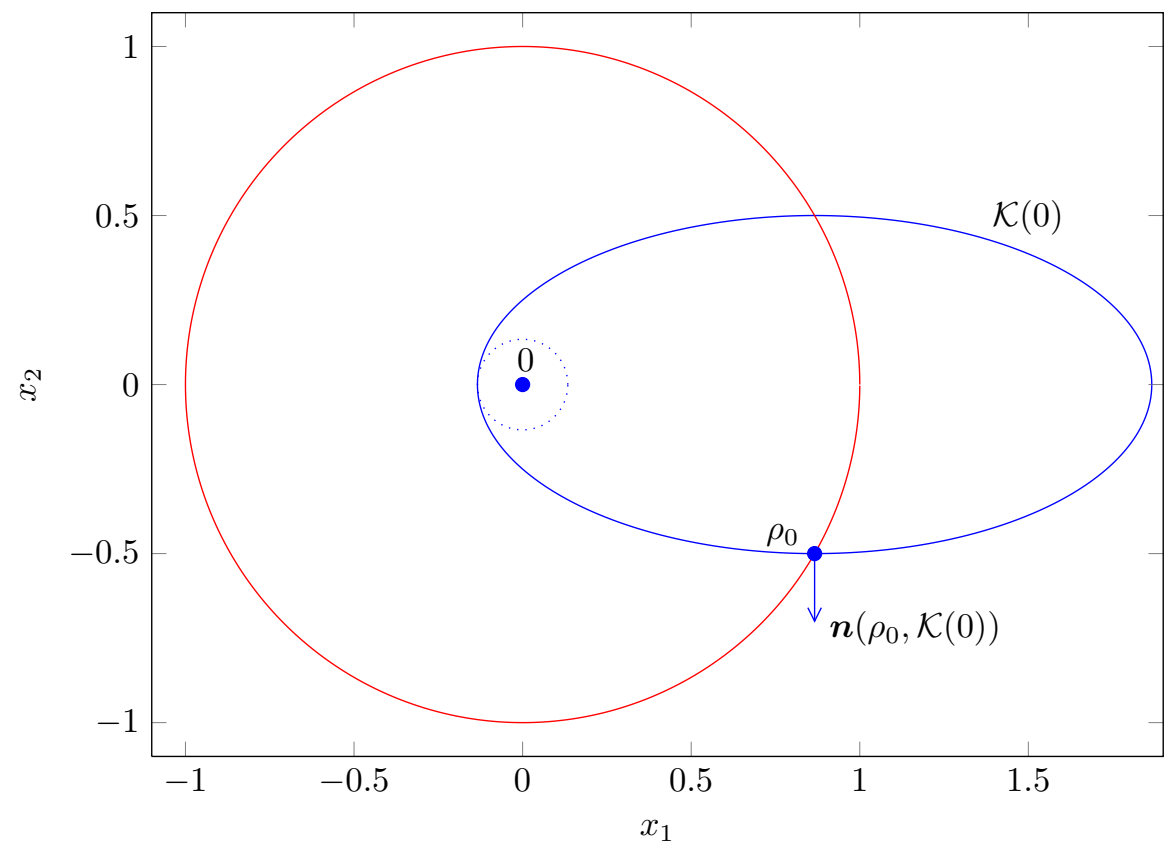

Figure 1: Construction of an example in dimension 2.

The solution $\hat{\boldsymbol{\rho}}$ is not elastic as $\frac{d}{d t}(\mathcal{L}(t) \hat{\boldsymbol{\rho}}(t)) \neq 0$. Since $\hat{\boldsymbol{\rho}}$ is periodic, it does not converge towards any elastic solution. We are therefore in a situation where Conditions 1-3 are all satisfied but not every solution of (15) converges towards an elastic solution.

Some complementing results are shown in Fig. 2. In that Figure are represented the trajectories of $\boldsymbol{\rho}$ starting from two different initial conditions (labelled as $A$ and $B$ ). Those trajectories are obtained by solving Eq. (15) numerically. The periodic solution $\hat{\rho}$ is shown in red in Figs. 1-2. Numerical computations indicate that solutions of (15) either converge towards an elastic solution (see point $A$ in Fig. 2) or are attracted towards the limit cycle $\hat{\boldsymbol{\rho}}$ (see point $B$ in Fig. 2). Convergence towards an elastic solution is observed only if the initial state is close enough to 0 . Such behavior fundamentally differs from the case of time-independent elastic moduli. In that last case, it is known that, for periodic variations of $\mathcal{K}(t)$, the asymptotic solutions are either all elastic or all inelastic. In other words, periodic elastic solutions and periodic inelastic solutions cannot coexist (Halphen, 1976; Wesfreid, 1981).

For what is to follow, the most important conclusion is that, when the elastic moduli depend on time, the Conditions 1-3 introduced in Sec. 3 are not sufficient to ensure that all solutions converge towards an elastic response. We prove that shakedown theorems can still be obtained for time-dependent elastic moduli. A central result is a general bound on the variations of the elastic energy, Eq. (50), that is established in the next section.

\section{Variation of the elastic energy}

Let us fix some notations to be used in the remainder of this paper: 


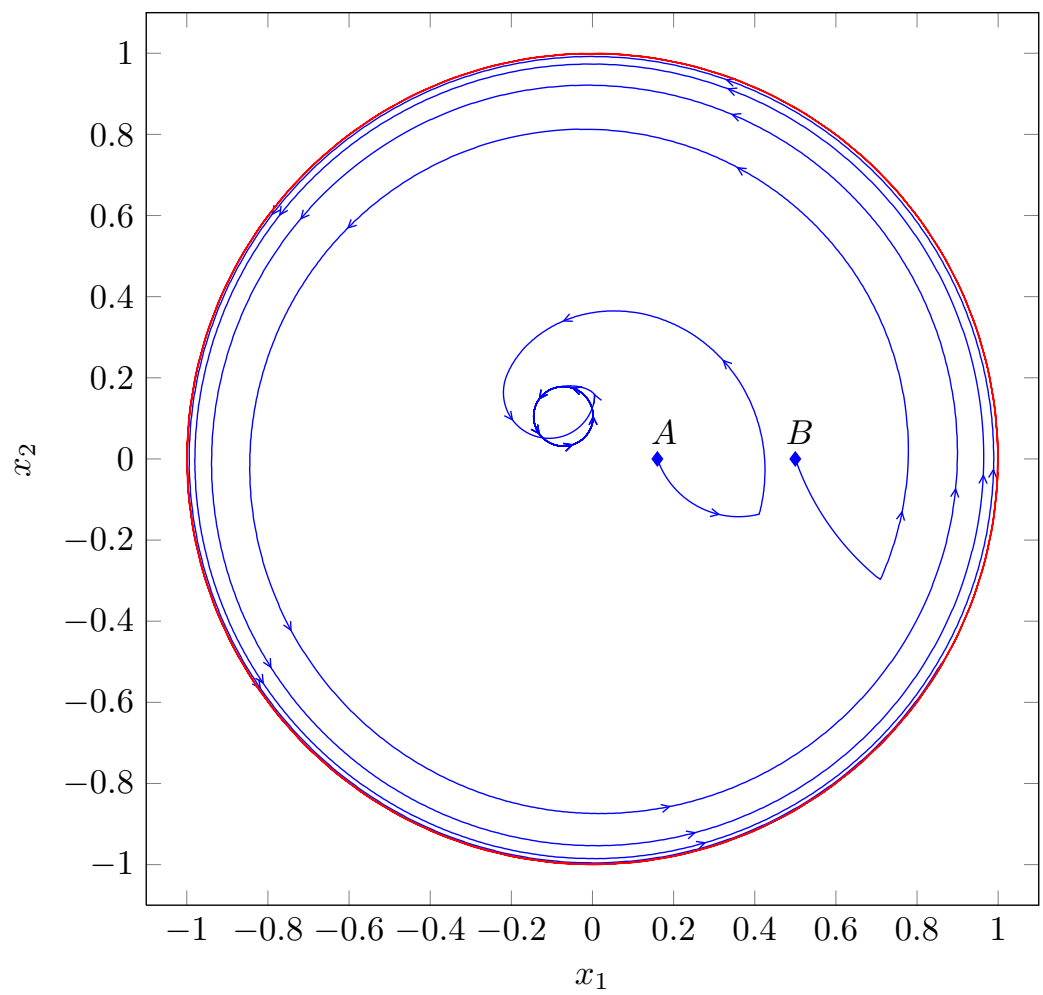

Figure 2: Elastic (in blue) and inelastic (in red) limit cycles for an example in dimension 2.

- $\mu(t)$ denotes an arbitrary non-negative function such that $\inf _{t} \mu(t)>0$. We set

$$
\mathcal{L}_{0}(t)=\mathcal{L}(t) / \mu(t), \mathcal{M}_{0}(t)=\mathcal{L}_{0}^{-1}(t)=\mu(t) \mathcal{M}(t) .
$$

with $\mathcal{M}(t)=\mathcal{L}(t)^{-1}$.

- Condition 3 is assumed to be satisfied for some elastic solution $\boldsymbol{\rho}^{*}(t)$ (on $[\tau,+\infty[$ ) and some $r>0$. The reason why we favor Condition 3 over Conditions 1-2 is that it seems to be the minimal requirement for conclusive results to be obtained. In finite dimension, we note that the fulfillment of Condition 1 or 2 automatically implies the fulfillment of Condition 3.

- Let $\boldsymbol{\rho}(t)$ be an arbitrary solution of (15). Setting $\boldsymbol{\tau}(t)=\mu(t)\left(\boldsymbol{\rho}(t)-\boldsymbol{\rho}^{*}(t)\right)$, the positive function $f$ defined as

$$
f(t)=\frac{1}{2}\left\langle\boldsymbol{\tau}(t), \mathcal{L}_{0}(t) \boldsymbol{\tau}(t)\right\rangle .
$$

is referred to as the elastic energy (of the stress field $\boldsymbol{\tau}(t)$ for the elastic operator $\mathcal{L}_{0}(t)$ ).

- The elastic moduli $\boldsymbol{L}(\boldsymbol{x}, t)$ are assumed to remain bounded, i.e. there exists some non-negative constants $\alpha$ et $\beta$ such that

$$
\alpha \leq \frac{\boldsymbol{\sigma}: \boldsymbol{L}(\boldsymbol{x}, t): \boldsymbol{\sigma}}{\boldsymbol{\sigma}: \boldsymbol{\sigma}} \leq \beta \text { for all } \boldsymbol{\sigma} \neq 0 .
$$


- The operator norm of $\mathcal{L}(t)$ is denoted by $\|\mathcal{L}(t)\|$, i.e.

$$
\|\mathcal{L}(t)\|=\sup _{\boldsymbol{\rho}^{\prime} \in \mathcal{H},\left\|\boldsymbol{\rho}^{\prime}\right\|=1}\left\|\mathcal{L}(t) \boldsymbol{\rho}^{\prime}\right\| .
$$

For latter reference, we note that Eq. (28) implies

$$
\alpha \leq\|\mathcal{L}(t)\| \leq \beta .
$$

The aim of this section is to obtain a bound on the variation $f(b)-f(a)$, where $a$ and $b$ are any given times such that $\tau \leq a \leq b$. We have

$$
\dot{f}(t)=\left\langle\boldsymbol{\tau}(t), \mathcal{L}_{0}(t) \dot{\boldsymbol{\tau}}(t)\right\rangle+\frac{1}{2}\left\langle\boldsymbol{\tau}(t), \dot{\mathcal{L}}_{0}(t) \boldsymbol{\tau}(t)\right\rangle .
$$

From (15) there exists $\boldsymbol{\eta}(t) \in \mathcal{H} \cap \partial I_{\mathcal{K}_{(t)}}(\boldsymbol{\rho}(t))$ such that

$$
\frac{d}{d t}(\mathcal{L}(t) \boldsymbol{\rho}(t))=-\boldsymbol{\eta}(t) .
$$

Since $d\left(\mathcal{L}(t) \boldsymbol{\rho}^{*}(t)\right) / d t=0$, we have equivalently

$$
\frac{d}{d t}\left(\mathcal{L}(t)\left(\boldsymbol{\rho}(t)-\boldsymbol{\rho}^{*}(t)\right)=-\boldsymbol{\eta}(t) .\right.
$$

Eq. (33) can be rewritten as $d\left(\mathcal{L}_{0}(t) \boldsymbol{\tau}(t)\right) / d t=-\boldsymbol{\eta}(t)$, i.e.

$$
\mathcal{L}_{0}(t) \dot{\boldsymbol{\tau}}(t)+\dot{\mathcal{L}}_{0}(t) \boldsymbol{\tau}(t)=-\boldsymbol{\eta}(t) .
$$

Substituting in (31) gives

$$
\dot{f}(t)=-\langle\boldsymbol{\tau}(t), \boldsymbol{\eta}(t)\rangle-\frac{1}{2}\left\langle\boldsymbol{\tau}(t), \dot{\mathcal{L}}_{0}(t) \boldsymbol{\tau}(t)\right\rangle .
$$

The rate of change $\dot{f}(t)$ of the elastic energy thus results from the contribution of two terms. The first term $-\langle\boldsymbol{\tau}(t), \boldsymbol{\eta}(t)\rangle$ is negative and directly associated with the plastic dissipation. The second term $-\left\langle\boldsymbol{\tau}(t), \dot{\mathcal{L}}_{0}(t) \boldsymbol{\tau}(t)\right\rangle / 2$ results from the time-dependence of the elastic moduli, and can be either positive or negative. Because of that term, $f$ is not necessarily decreasing with time and cannot be used as a Lyapunov function for the evolution problem (15). However, the function $f$ can still be used to study the asymptotic behaviour, as we shall see. The crucial point is to carefully study how the two terms in (34) compete against each other. To that purpose, we first note from (24) that

$$
\langle\boldsymbol{\tau}(t), \boldsymbol{\eta}(t)\rangle=\mu(t)\left\langle\boldsymbol{\rho}(t)-\boldsymbol{\rho}^{*}(t), \boldsymbol{\eta}(t)\right\rangle \geq r(\inf \mu)\|\boldsymbol{\eta}(t)\| .
$$

Replacing in (34) and integrating on the time interval $[a, b]$, we obtain

$$
f(b)-f(a) \leq-r(\inf \mu) \int_{a}^{b}\|\boldsymbol{\eta}\| d t+\int_{a}^{b} H(t) d t
$$

with

$$
H(t)=-\frac{1}{2}\left\langle\boldsymbol{\tau}(t), \dot{\mathcal{L}}_{0}(t) \boldsymbol{\tau}(t)\right\rangle .
$$


In order to compare the two terms on the right-hand side of (35), we aim at bounding the integral $\int_{a}^{b} H(t) d t$ in terms of $\int_{a}^{b}\|\boldsymbol{\eta}\| d t$. The first step is to rewrite $H(t)$ in a form where $\boldsymbol{\eta}$ appears explicitly. To that purpose, we set

$$
\boldsymbol{T}(t)=-\int_{a}^{t} \boldsymbol{\eta}(s) d s
$$

so that the integration of (33) gives

$$
\boldsymbol{\tau}(t)=\mathcal{L}_{0}^{-1}(t)(\boldsymbol{s}+\boldsymbol{T}(t))
$$

where

$$
\boldsymbol{s}=\mathcal{L}(a)\left(\boldsymbol{\rho}(a)-\boldsymbol{\rho}^{*}(a)\right)
$$

is independent on $t$. Substituting the expression (38) in (36) provides

$$
H(t)=-\frac{1}{2}\left\langle\mathcal{L}_{0}^{-1}(t)(\boldsymbol{T}(t)+\boldsymbol{s}), \dot{\mathcal{L}}_{0}(t) \mathcal{L}_{0}^{-1}(t)(\boldsymbol{T}(t)+\boldsymbol{s})\right\rangle .
$$

Using the fact that $\mathcal{L}_{0}(t)$ is self-adjoint, we have

$$
H(t)=-\frac{1}{2}\left\langle(\boldsymbol{T}(t)+\boldsymbol{s}), \mathcal{L}_{0}^{-1}(t) \dot{\mathcal{L}}_{0}(t) \mathcal{L}_{0}^{-1}(t)(\boldsymbol{T}(t)+\boldsymbol{s})\right\rangle .
$$

Observing that $\mathcal{L}_{0}^{-1}(t) \dot{\mathcal{L}}_{0}(t) \mathcal{L}_{0}^{-1}(t)=-\dot{\mathcal{M}}_{0}(t)$, we find

$$
H(t)=\frac{1}{2}\left\langle(\boldsymbol{T}(t)+\boldsymbol{s}), \dot{\mathcal{M}}_{0}(t)(\boldsymbol{T}(t)+\boldsymbol{s})\right\rangle .
$$

In view of the definition (37) of $\boldsymbol{T}(t)$, Eq. (41) can be seen to relate $H(t)$ to the time integral of $\boldsymbol{\eta}$. Since $s$ does not depend on $t$, the integration of (41) on the time interval $[a, b]$ gives

$$
\int_{a}^{b} H(t) d t=\frac{1}{2}\left\langle\boldsymbol{s},\left(\mathcal{M}_{0}(b)-\mathcal{M}_{0}(a)\right) s\right\rangle+\frac{1}{2} \int_{a}^{b}\left\langle\boldsymbol{T}(t), \dot{\mathcal{M}}_{0}(t)(\boldsymbol{T}(t)+2 s)\right\rangle d t .
$$

The next step is to bound the integral on the right-hand side of (42). Using the Cauchy-Schwartz inequality and the definition (29) of the norm operator, we obtain

$$
\left\langle\boldsymbol{T}(t), \dot{\mathcal{M}}_{0}(t)(\boldsymbol{T}(t)+2 s)\right\rangle \leq\|\boldsymbol{T}(t)\| \cdot\left\|\dot{\mathcal{M}}_{0}(\boldsymbol{T}(t)+2 s)\right\| \leq\|\boldsymbol{T}(t)\| \cdot\left\|\dot{\mathcal{M}}_{0}(t)\right\| \cdot\|\boldsymbol{T}(t)+2 s\| .
$$

Let $M$ and $C$ be positive constants such that

$$
\begin{gathered}
\|\mathcal{L}(t)\| \leq C \text { for all } t \geq \tau \\
\left\|\boldsymbol{\rho}^{\prime}\right\| \leq M \text { for all } t \geq \tau \text { and } \boldsymbol{\rho}^{\prime} \in \mathcal{K}(t) .
\end{gathered}
$$

The property (30) ensures that a value of $C$ satisfying (44) can be found. The existence of a constant $M$ satisfying (45) rests on the assumption that the set $\mathcal{K}(t)$ is bounded, which requires some comments. If the elasticity domain $\mathcal{C}(\boldsymbol{x}, t)$ and the elastic response $\boldsymbol{\sigma}^{E}(t)$ are bounded, then the set $\mathcal{K}(t)$ can readily be seen to be bounded in $\mathcal{H}$, independently on time $t$. In many situations, however, only the set of deviatoric tensors in $\mathcal{C}(\boldsymbol{x}, t)$ is bounded. This notably occurs for the Von Mises and the Tresca criteria. In such a situation, it can be proved that $\mathcal{K}(t)$ is still bounded 
provided that $\partial \Omega_{t} \neq \emptyset$, i.e. provided that tractions are specified on some points on the boundary of the domain (Mercier, 1977; Halphen, 1976). have

From the definition (39) of $s$ and the distinctive properties (44) of the constants $C$ and $M$, we

$$
\|\boldsymbol{s}\| \leq C\left\|\boldsymbol{\rho}(0)-\boldsymbol{\rho}^{*}(0)\right\| \leq 2 C M .
$$

In a similar fashion, we deduce from (32) that

$$
\|\boldsymbol{T}(t)\|=\|\mathcal{L}(t) \boldsymbol{\rho}(t)-\mathcal{L}(0) \boldsymbol{\rho}(0)\| \leq C(\|\boldsymbol{\rho}(t)\|+\|\boldsymbol{\rho}(0)\|) \leq 2 C M
$$

and therefore that

$$
\|\boldsymbol{T}(t)+2 \boldsymbol{s}\| \leq\|\boldsymbol{T}(t)\|+2\|\boldsymbol{s}\| \leq 6 C M .
$$

Eq. (37) also provides the following bound on $\boldsymbol{T}(t)$ :

$$
\|\boldsymbol{T}(t)\|=\left\|\int_{a}^{t} \boldsymbol{\eta}(s) d s\right\| \leq \int_{a}^{t}\|\boldsymbol{\eta}(s)\| d s \leq \int_{a}^{b}\|\boldsymbol{\eta}(s)\| d s .
$$

Substituting (46-47) in (43) and using (42), we obtain

$$
\int_{a}^{b} H(t) d t \leq \frac{1}{2}\left\langle\boldsymbol{s},\left(\mathcal{M}_{0}(T)-\mathcal{M}_{0}(0)\right) s\right\rangle+3 C M \gamma(a, b) \int_{a}^{b}\|\boldsymbol{\eta}(s)\| d s
$$

where

$$
\gamma(a, b)=\int_{a}^{b}\left\|\dot{\mathcal{M}}_{0}(t)\right\| d t=\int_{a}^{b}\|\mu(t) \dot{\mathcal{M}}(t)+\dot{\mu}(t) \mathcal{M}(t)\| d t
$$

Replacing (48) in (35), we finally arrive at

$$
f(b)-f(a) \leq \frac{1}{2}\left\langle\boldsymbol{s},\left(\mathcal{M}_{0}(b)-\mathcal{M}_{0}(a)\right) s\right\rangle+(3 C M \gamma(a, b)-r(\inf \mu)) \int_{a}^{b}\|\boldsymbol{\eta}(t)\| d t .
$$

Eq. (50) gives an upper bound on the variation of the elastic energy on the time interval $[a, b]$. The right-hand side of (50) can be broken up as the sum of three terms that we analyze separately. The term $\frac{1}{2}\left\langle\boldsymbol{s},\left(\mathcal{M}_{0}(b)-\mathcal{M}_{0}(a)\right) s\right\rangle$ corresponds to the variation due to the time fluctuations of the elastic moduli alone. In particular, if the evolution is elastic on $[a, b]$, then the right-hand side of $(50)$ reduces to $\frac{1}{2}\left\langle\boldsymbol{s},\left(\mathcal{M}_{0}(b)-\mathcal{M}_{0}(a)\right) s\right\rangle$ and the bound (50) is sharp. The term $-r(\inf \mu) \int_{a}^{b}\|\boldsymbol{\eta}(t)\| d t$ in (50) is related to the dissipated energy and does not depend explicitly on the elastic moduli. In particular, in the case where $\boldsymbol{M}_{0}(t)$ is time-independent, then the right-hand side of (50) reduces to $-r(\inf \mu) \int_{a}^{b}\|\boldsymbol{\eta}(t)\| d t$. The last term $3 C M \gamma(a, b) \int_{a}^{b}\|\boldsymbol{\eta}(t)\| d t$ accounts for the coupling between plastic yielding and the time-variations of the elastic moduli. It is helpful to have a geometric interpretation of the quantity $\gamma(a, b)$ defined in (49): picturing $\mathcal{M}_{0}(t)$ as a moving point in the space of linear operators on $\mathcal{H}, \gamma(a, b)$ corresponds to the distance covered by $\boldsymbol{\mathcal { M }}_{0}(t)$ between time $a$ and time $b$ (Fig. 3).

Eq. (50) is the main result that allows us to study convergence of solutions to (15). As a first illustration, consider the situation where $\mathcal{M}(t)$ converges towards a limit $\mathcal{M}^{\infty}$ as $t \longrightarrow \infty$, with $\int_{0}^{\infty}\|\dot{\mathcal{M}}(t)\| d t<\infty$ (Fig. 3). In such condition, there exists a time $\kappa$ such that

$$
\int_{\kappa}^{T}\|\dot{\mathcal{M}}(t)\| d t<\frac{r}{6 C M}
$$




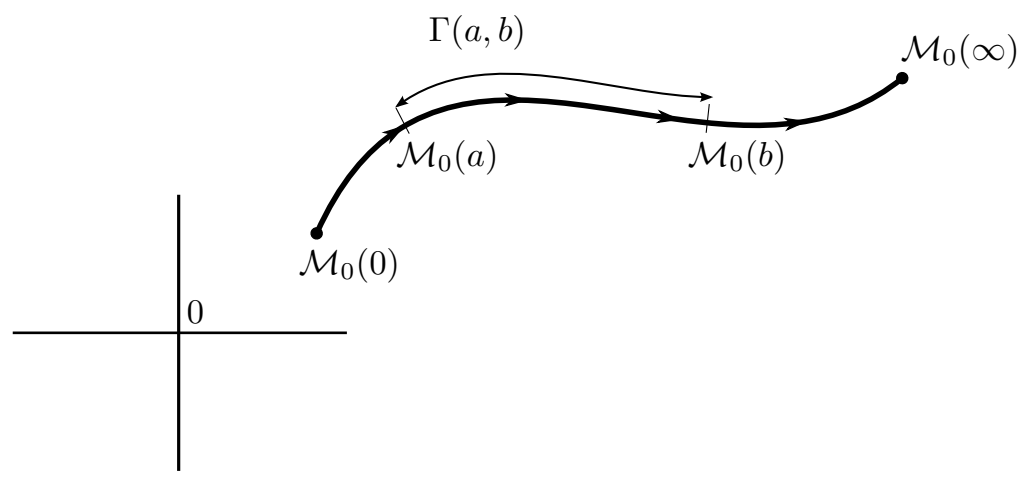

Figure 3: Geometric interpretation of $\gamma(a, b)$.

for any $T \geq \kappa$. Choosing $\mu(t)=1$ and using (50) on the time interval $[\kappa, T]$, we obtain

$$
f(T)-f(\kappa) \leq \frac{1}{2}\langle\boldsymbol{s},(\boldsymbol{M}(T)-\mathcal{M}(\kappa)) s\rangle-\frac{r}{2} \int_{\kappa}^{T}\|\boldsymbol{\eta}(s)\| d s .
$$

Therefore, recalling that $f \geq 0$,

$$
r \int_{\kappa}^{T}\|\boldsymbol{\eta}(t)\| d t \leq\langle\boldsymbol{s},(\boldsymbol{M}(T)-\mathcal{M}(\kappa)) \boldsymbol{s}\rangle+2 f(\kappa) .
$$

The right-hand side of (51) converges towards $\left\langle\boldsymbol{s},\left(\mathcal{M}^{\infty}-\mathcal{M}(\kappa)\right) \boldsymbol{s}\right\rangle+2 f(\kappa)$ as $T \longrightarrow \infty$. It follows that $\int_{\kappa}^{+\infty}\|\boldsymbol{\eta}(s)\| d s<\infty$ and therefore that $\int_{0}^{T} \boldsymbol{\eta}(s) d s$ converges to a limit as $T \rightarrow \infty$. Setting $\boldsymbol{\eta}_{\infty}=\mathcal{L}(0) \boldsymbol{\rho}(0)-\int_{0}^{\infty} \boldsymbol{\eta}(s) d s$, we obtain from (32) that

$$
\mathcal{L}(t) \boldsymbol{\rho}(t) \longrightarrow \boldsymbol{\eta}_{\infty} \text { as } t \longrightarrow \infty .
$$

The conclusion is that any solution of (15) asymptotically behaves as $\boldsymbol{\mathcal { M }}(t) \boldsymbol{\eta}_{\infty}$ for some timeindependent $\boldsymbol{\eta}_{\infty}$, i.e. that shakedown occurs.

The case of time periodic variations of the elastic moduli, which is of practical importance, is studied in detail in the next section.

\section{Periodic variations of the elastic moduli}

\subsection{Sufficient condition for shakedown}

In this section we assume that $\mathcal{L}(t)$ is $T$-periodic, i.e. of period $T$. Let $\mu(t)$ be a nonnegative $T$-periodic function, so that $\mathcal{M}_{0}(t)$ is also $T$-periodic. Set

$$
m=3 C M \gamma(0, T)-r \inf \mu
$$

and assume that $m<0$. Using (50) on the time interval $[n T,(n+1) T]$ (with $n \in \mathbb{N}$ ) we obtain

$$
f((n+1) T)-f(n T) \leq m \int_{n T}^{(n+1) T}\|\boldsymbol{\eta}(t)\| d t
$$


where the properties $\mathcal{M}_{0}(n T)=\mathcal{M}_{0}((n+1) T)$ and $\gamma(n T,(n+1) T)=\gamma(0, T)$ have been used. Summing (52) over $n=0, \cdots, N-1$ gives

$$
\int_{0}^{N T}\|\boldsymbol{\eta}(t)\| d t \leq-\frac{f(0)}{m}
$$

It follows that $\int_{0}^{\infty}\|\boldsymbol{\eta}(t)\| d t<\infty$. The same reasoning as used previously shows that any solution of (15) asymptotically behaves as $\boldsymbol{\mathcal { M }}(t) \boldsymbol{\eta}_{\infty}$ for some time-independent $\boldsymbol{\eta}_{\infty}$. We can now state the following theorem:

Shakedown theorem 1 (time-periodic elastic moduli). If

(i) there exists $r>0$ and an elastic solution $\boldsymbol{\rho}^{*}(t)$ (on a time interval $[\tau,+\infty[$ ) such that $\left(\mathcal{H} \cap B\left(\boldsymbol{\rho}^{*}(t), r\right)\right) \subset \mathcal{K}(t)$ for all $t \geq \tau$;

(ii) the elastic moduli are such that

$$
\frac{\gamma(0, T)}{\inf \mu}<\frac{r}{3 C M}
$$

for some non-negative $T$-periodic function $\mu(t)$;

then the residual stress converges towards an elastic solution, whatever the initial state is.

Condition $(i)$ is simply a restatement of Condition 3 discussed before and does not call for any special comment. We simply mention that, if $\mathcal{H}$ is of finite dimension, the fulfillment of the more familiar Condition 1 ensures that $(i)$ is satisfied. In view of the counterexample given in Sec. 4, it is not surprising to find additional requirement complementing $(i)$ in Theorem 1 . The condition (ii) that is obtained sets a constraint on the time variations of the elastic moduli. Several remarks are in order regarding condition (ii).

Remark 1: provided condition $(i)$ is satisfied, we note that condition $(i i)$ is automatically fulfilled if $\mathcal{M}(t)$ varies as

$$
\mathcal{M}(t)=\lambda(t) \mathcal{M}_{0}
$$

where $\lambda(t)$ is a non negative scalar and $\mathcal{M}_{0}$ is a time-independent operator. Choosing $\mu(t)=1 / \lambda(t)$ in (26), we obtain indeed that $\gamma(0, T)=0$. Eq. (53) corresponds to the situation where $\mathcal{M}(t)$ describes a radial path in the space of linear operators on $\mathcal{H}$, i.e $\mathcal{M}(t)$ moves on a half-line passing through the origin. In that special case, an alternate (and more direct) proof of Theorem 1 can be obtained (see Appendix A).

Remark 2: as observed previously, $\gamma(0, T)$ is the length of the closed curve described by $\mathcal{M}_{0}(t)$ and thus remains unchanged if a time rescaling is performed. Condition (ii) is therefore rateindependent, which is in line with the rate-independent nature of the elastic-perfectly plastic behaviour.

Remark 3: as mentioned in the introduction, incremental analysis has been used by Halphen and di Domizio (2005); Hasbroucq et al. (2010, 2012) to study the asymptotic behaviour of some specific mechanical systems with time-dependent elastic moduli. For the examples considered by those authors, condition $(i)$ was found to be sufficient for shakedown to occur independently on the initial state. It is interesting at this point to give more details on the systems that were studied. In the plate problem considered by Hasbroucq et al. (2012), the elasticity tensor $\boldsymbol{L}(x, t)$ is taken as 
isotropic with a constant Poisson's ratio $\nu$ and a spatially homogeneous Young's modulus $E(t)$. In such condition, the tensor $\boldsymbol{L}(\boldsymbol{x}, t)$ can be written as $\boldsymbol{L}(\boldsymbol{x}, t)=E(t) \boldsymbol{L}_{0}$ where $\boldsymbol{L}_{0}$ is constant (both in space and time). It follows from the definition (13) that the corresponding operator $\mathcal{L}(t)$ is of the form (53). In the two-bar system considered in Hasbroucq et al. (2010), the Young's modulus is not spatially homogeneous but the space $\mathcal{H}$ of self-stresses is of dimension 1 . Therefore, the linear operator $\mathcal{L}(t): \mathcal{H} \mapsto \mathcal{H}$ is also of the radial form (53). Similar remarks hold for the examples studied by Halphen and di Domizio (2005). Therefore, there is no contradiction between Theorem 1 and the results of Halphen and di Domizio (2005); Hasbroucq et al. (2010, 2012): for all the examples studied by the authors, it turns out that $\mathcal{L}(t)$ is of the form (53), in which case condition (ii) is automatically satisfied if condition $(i)$ is.

Remark 4: in condition $(i i)$, the function $\mu(t)$ acts as an arbitrary parameter. We note that multiplying $\mu$ by a non-negative constant does not change the value of $\gamma(0, T) / \inf \mu$. Further comments on $\mu(t)$ are given in the following subsection.

\subsection{Choice of $\mu(t)$}

Consider a given periodic function $\mathcal{L}(t)$ and assume that condition $(i)$ in Theorem 1 is satisfied. In order to check condition (ii), a natural choice is to take $\mu(t)=1$. That choice, however, is not necessarily optimal. Consider for instance the case (53) of radial variations. Taking $\mu(t)=1$, we obtain from Theorem 1 that shakedown occurs provided that

$$
\int_{0}^{T}|\dot{\lambda}(t)| d t<\frac{r}{3 C M\left\|\mathcal{M}_{0}\right\|}
$$

If we take $\mu(t)=1 / \lambda(t), \gamma(0, T)$ vanishes and we obtain from Theorem 1 that shakedown occurs

for all values of $\int_{0}^{T}|\dot{\lambda}(t)| d t$, as noticed in Remark 1 above. This example shows that all choices of $\mu(t)$ are not equivalent, which in turn raises the question of what is the best choice for $\mu(t)$. A simple prescription that we adopt in this paper is

$$
\mu(t)=\frac{1}{\operatorname{tr} \mathcal{M}(t)} .
$$

In the particular case of radial variations, Eq. (54) gives $\gamma(0, T)=0$. We note that the prescription (54) is not necessarily optimal in all cases and that other choices are possible. In principle, the optimal value of $\mu$ is obtained by minimizing $\gamma(0, T) / \inf \mu$, i.e. by solving

$$
\inf _{\mu(t)>0 \mid \mu(0)=\mu(T)} \frac{1}{\inf \mu} \int_{0}^{T}\|\mu(t) \dot{\mathcal{M}}(t)+\dot{\mu}(t) \mathcal{M}(t)\| d t .
$$

However, notably because of the non differentiability of the norm operator $\|\cdot\|$, solving (55) seems to be difficult in general and is not further addressed in this paper.

\subsection{Expression in terms of local variations of the elastic moduli}

The practical application of Theorem 1 is hampered by the fact that the expression of the linear operator $\mathcal{M}(t)$ is needed (so as to determine $\gamma(0, T)$ as well as the constant $C$ in (44)). Assuming the space $\mathcal{H}$ to be of finite dimension, we derive in this section a shakedown theorem which is expressed only in terms of the local variations of the elastic moduli $\boldsymbol{L}(\boldsymbol{x}, t)$, thus sparing the calculation of $\mathcal{M}(t)$. 
Let $\lambda_{1}(t), \cdots, \lambda_{n}(t)$ be the eigenvalues of the symmetric positive operator $\mathcal{L}(t)$. It can be seen from (13) and (28) that $\alpha \leq \lambda_{i}(t) \leq \beta$ for $i=1, \cdots, n$. Since $\mathcal{M}(t)=\mathcal{L}^{-1}(t)$, we have

$$
\|\mathcal{M}(t)\|=\max _{i} \frac{1}{\lambda_{i}(t)} \leq \frac{1}{\alpha}
$$

We now bound $\gamma(0, T)=\int_{0}^{T}\|\mu(t) \dot{\mathcal{M}}(t)+\dot{\mu}(t) \mathcal{M}(t)\| d t$ in terms of local quantities. Using the identity $\dot{\mathcal{M}}+y \mathcal{M}=\mathcal{M}(-\dot{\mathcal{L}}+y \mathcal{L}) \mathcal{M}$, we have, for any $y$,

$$
\|\dot{\mathcal{M}}(t)+y \mathcal{M}(t)\| \leq\|\mathcal{M}(t)\|^{2} \cdot\|\dot{\mathcal{L}}(t)-y \mathcal{L}(t)\| \leq \frac{1}{\alpha^{2}}\|\dot{\mathcal{L}}(t)-y \mathcal{L}(t)\|
$$

Let $G(y, t)$ be defined by

$$
G(y, t)=\sup _{\boldsymbol{x} \in \Omega} \sup _{\boldsymbol{\sigma} \neq 0} \frac{|\boldsymbol{\sigma}:(\dot{\boldsymbol{L}}(\boldsymbol{x}, t)-y \boldsymbol{L}(\boldsymbol{x}, t)): \boldsymbol{\sigma}|}{\boldsymbol{\sigma}: \boldsymbol{\sigma}} .
$$

The function $G$ is determined by the local variations of $\boldsymbol{L}(\boldsymbol{x}, t)$. It can be seen from (13) that $\|\dot{\mathcal{L}}-y \mathcal{L}\| \leq G(y, t)$. Hence

$$
\gamma(0, T)=\int_{0}^{T} \mu(t)\left\|\dot{\mathcal{M}}(t)+\frac{\dot{\mu}(t)}{\mu(t)} \mathcal{M}(t)\right\| d t \leq \int_{0}^{T} \frac{\mu(t)}{\alpha^{2}} G\left(\frac{\dot{\mu}(t)}{\mu(t)}, t\right) d t .
$$

Using Theorem 1, we can state the following result:

\section{Shakedown theorem 2 (time-periodic elastic moduli). If}

(i) there exists $r>0$ and an elastic solution $\boldsymbol{\rho}^{*}(t)$ (on a time interval $[\tau,+\infty[$ ) such that $\left(\mathcal{H} \cap B\left(\boldsymbol{\rho}^{*}(t), r\right)\right) \subset \mathcal{K}(t)$ for all $t \geq \tau$;

(ii) the elastic moduli are such that

$$
\int_{0}^{T} \mu(t) G\left(\frac{\dot{\mu}(t)}{\mu(t)}, t\right) d t<\frac{r(\inf \mu) \alpha^{2}}{3 M \beta}
$$

for some non-negative $T$-periodic function $\mu(t)$;

then the residual stress field converges towards an elastic solution, whatever the initial state is.

In practice, Theorem 2 is expected to be easier to use than Theorem 1 because the calculation of the linear operator $\mathcal{L}(t)$ is avoided. It has to be noted, however, that the condition $(i i)$ set in Theorem 2 on the time variations of the elastic moduli is more restrictive than the condition $(i i)$ in Theorem 1.

As for Theorem 1, the question raises of how to chose the multiplier $\mu(t)$ so as to minimize the quantity $\left(\int_{0}^{T} \mu(t) G\left(\frac{\dot{\mu}(t)}{\mu(t)}\right) d t\right) / \inf \mu$. In that respect, we note that a simple prescription that mimics (54) is to take

$$
\mu(t)=\int_{\Omega} \operatorname{tr} \boldsymbol{L}(\boldsymbol{x}, t) d \omega
$$




\section{Illustrative example}

We use the theorems of Sec. 6 to study the mechanical system represented in Fig. 4. That system consists of three elastic-plastic bars which are connected to rigid blocks at both extremities. The rigid blocks are constrained to move only along the direction of the bars.

The system is at equilibrium under a constant force $F$, so that

$$
\sigma_{1}+\sigma_{2}+\sigma_{3}=F / A
$$

where $\sigma_{i}$ is the uniaxial stress in bar $i$ and $A$ is the area of the cross-sections of the bars. The geometric compatibility gives the relations

$$
\epsilon_{1}=\epsilon_{2}=\epsilon_{3}
$$

where $\epsilon_{i}$ is the uniaxial strain in bar $i$.

The constitutive law in each bar is written as

$$
\begin{aligned}
\epsilon_{i} & =\frac{\sigma_{i}}{E_{i}}+\epsilon_{i}^{p}+\epsilon_{i}^{\theta}, \\
\dot{\epsilon}_{i}^{p} & \in \partial I_{\left[-\sigma_{y}, \sigma_{y}\right]}\left(\sigma_{i}\right)
\end{aligned}
$$

where the Young's modulus $E_{i}$ and the thermal strain $\epsilon_{i}^{\theta}$ in each bar are $T$-periodic functions of time satisfying

$$
E_{1}(t)=E_{3}(t), \epsilon_{1}^{\theta}(t)=\epsilon_{3}^{\theta}(t) .
$$

The yield limit $\sigma_{y}$ is assumed to be independent on $t$.

Writing the stress state of the system as $\boldsymbol{\sigma}=\left(\sigma_{1}, \sigma_{2}, \sigma_{3}\right)$, the fictitious elastic response of the system is

$$
\boldsymbol{\sigma}^{E}(t)=\frac{\epsilon^{\theta}(t)}{g_{1}(t)+2 g_{2}(t)}(1,-2,1)+\frac{F}{A} \frac{1}{g_{1}(t)+2 g_{2}(t)}\left(g_{2}(t), g_{1}(t), g_{2}(t)\right)
$$

where $\epsilon^{\theta}(t)=\epsilon_{2}^{\theta}(t)-\epsilon_{1}^{\theta}(t)$ and $g_{i}(t)=1 / E_{i}(t)$.

The asymptotic behavior of the elastic-plastic three-bar structure is well known when the elastic moduli do not depend on time. In the particular case $E_{1}=E_{2}=E$ (where $E$ is a constant), Melan's theorem ensures that shakedown provided that

$$
\frac{\sigma^{\theta}}{\sigma_{y}} \leq \min \left(\frac{3}{2}, \frac{3}{4}\left(3-\frac{\sigma^{F}}{\sigma_{y}}\right)\right)
$$

with

$$
\sigma^{F}=\frac{|F|}{A}, \sigma^{\theta}=E \sup _{t}\left|\epsilon^{\theta}(t)\right| .
$$

In the following we investigate how those results are modified when the Young's moduli are timedependent. 


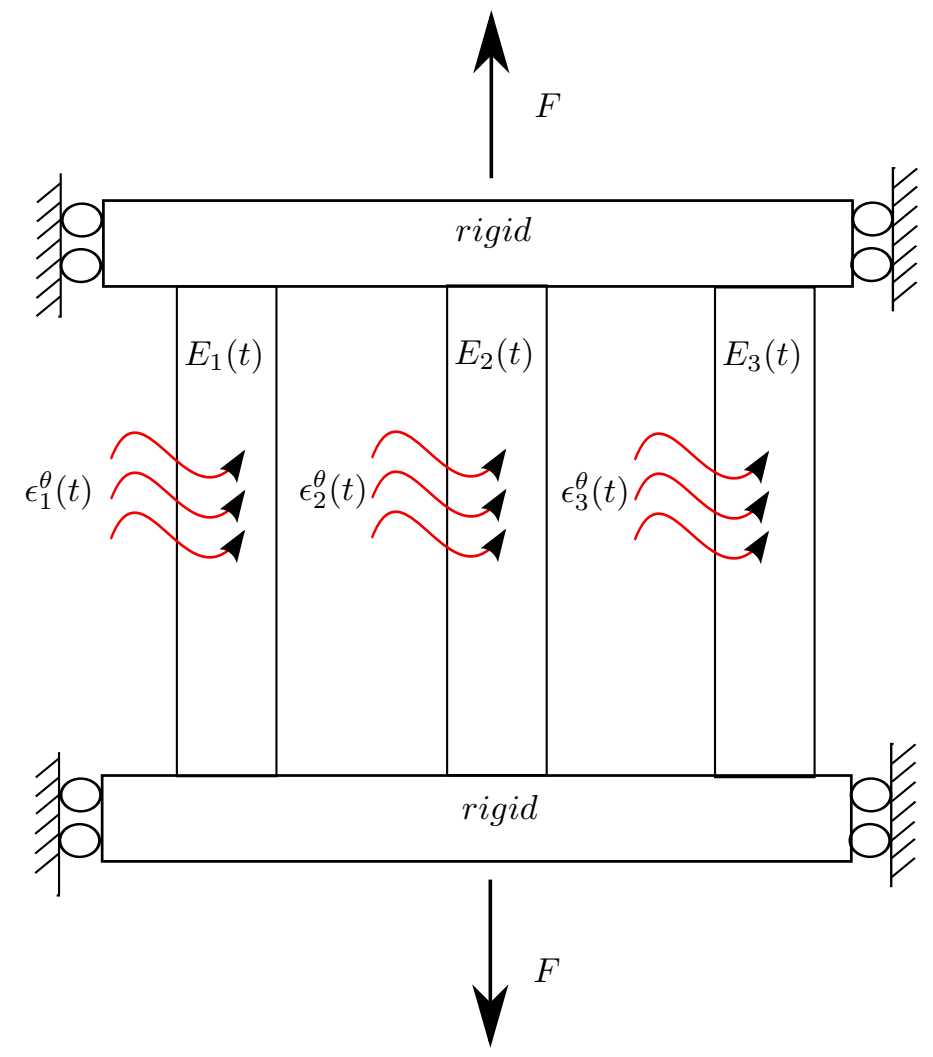

Figure 4: Three-bar structure.

\subsection{Stress-free evolutions}

For the example considered, the vectorial space $\mathcal{E}$ of stress fields identifies with $\mathbb{R}^{3}$ and is equipped with the scalar product $\left\langle\boldsymbol{\sigma}, \boldsymbol{\sigma}^{\prime}\right\rangle=\sum_{i=1}^{3} \sigma_{i} \sigma_{i}^{\prime}$. From (57) the space $\mathcal{H}$ of residual stress fields is the two-dimensional subspace of $\mathcal{E}$ defined by the equation $\sigma_{1}+\sigma_{2}+\sigma_{3}=0$. An orthonormal basis of $\mathcal{H}$ is $\left(\boldsymbol{e}^{1}, \boldsymbol{e}^{2}\right)$ with $\boldsymbol{e}^{1}=(1,0,-1) / \sqrt{2}$ and $\boldsymbol{e}^{2}=(-1,2,-1) / \sqrt{6}$. The coordinates in that basis are denoted by $\left(x_{1}, x_{2}\right)$.The convex set $\mathcal{K}_{0}$ of plastically admissible stress fields is the cube in $\mathbb{R}^{3}$ defined by

$$
\mathcal{K}_{0}=\left\{\left(\sigma_{1}, \sigma_{2}, \sigma_{3}\right):\left|\sigma_{i}\right| \leq \sigma_{y} \text { for } i=1,2,3\right\} .
$$

We first focus on the case $\sigma^{F}=0$, which allows for some closed-form expressions to be obtained. In such situation, the convex set $\mathcal{K}(t)$ can be written as

$$
\mathcal{K}(t)=-\boldsymbol{\sigma}^{E}(t)+\mathcal{K}_{0} \cap \mathcal{H}=\sqrt{6} \frac{\epsilon^{\theta}(t)}{g_{1}(t)+2 g_{2}(t)} \boldsymbol{e}_{2}+\mathcal{K}_{0} \cap \mathcal{H}
$$

where $\mathcal{K}_{0} \cap \mathcal{H}$ is the hexagonal domain defined by

$$
\left|x_{2}\right| \leq \frac{\sqrt{6} \sigma_{y}}{2},\left|\sqrt{3} x_{1} \pm x_{2}\right| \leq \sqrt{6} \sigma_{y}
$$




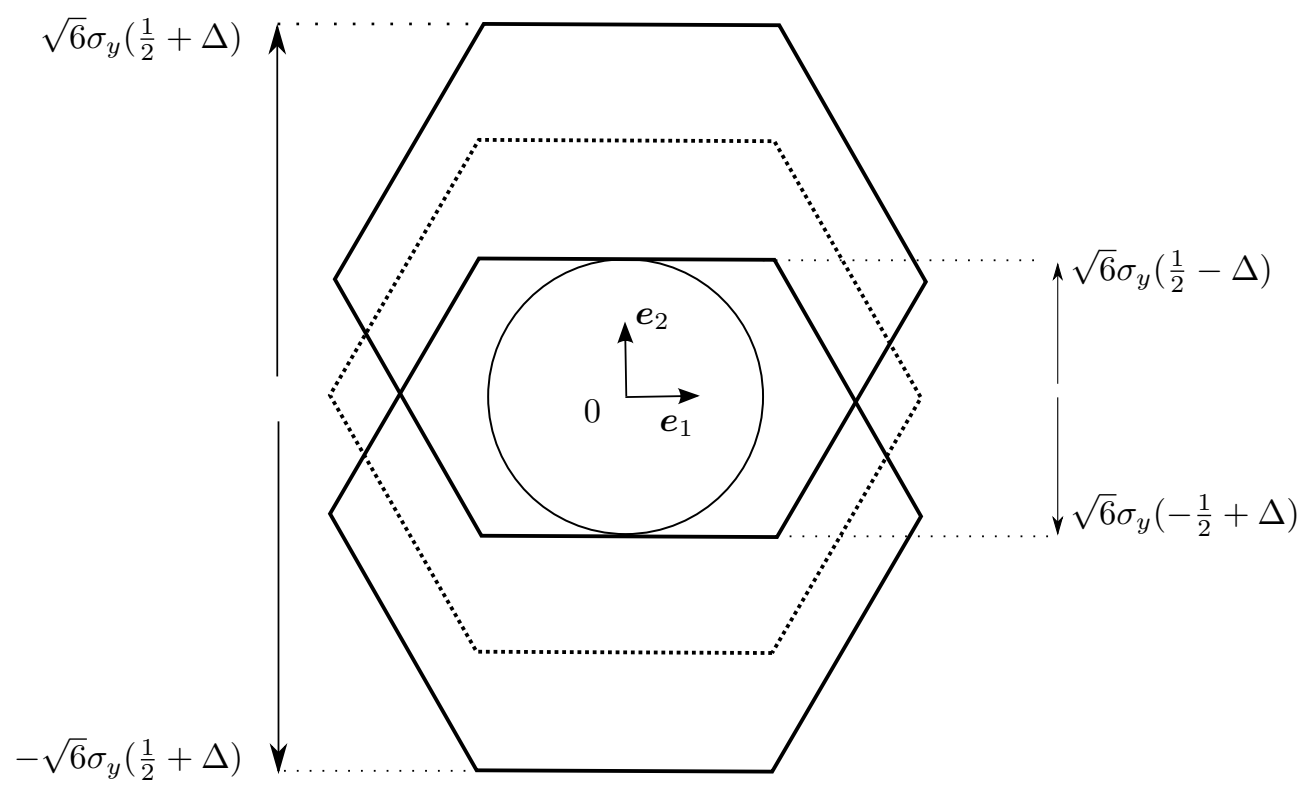

Figure 5: the moving set $\mathcal{K}(t)$ in the case $F=0$.

As $t$ varies, $\mathcal{K}(t)$ is translating along the $\boldsymbol{e}_{2}$ axis, as represented in Fig. 5.

It can easily be verified that $\sup _{\boldsymbol{\rho} \in \mathcal{K}(t)}\|\boldsymbol{\rho}\|$ is reached at the corner of $\mathcal{K}(t)$ defined by

$$
x_{1}=\sqrt{2} \frac{\sigma_{y}}{2}, x_{2}=\operatorname{sgn}\left(\epsilon^{\theta}(t)\right) \sqrt{6} \frac{\sigma_{y}}{2}+\frac{\sqrt{6} \epsilon^{\theta}(t)}{g_{1}(t)+2 g_{2}(t)} .
$$

The constant $M$ in (45) can thus be taken as

$$
M=\sigma_{y} \sqrt{2+6 \Delta^{2}+6 \Delta}
$$

with

$$
\Delta=\sup _{t} \frac{\left|\epsilon^{\theta}(t)\right|}{\sigma_{y}\left(g_{1}(t)+2 g_{2}(t)\right)} .
$$

As long as $\Delta \leq 1 / 2$, it can be observed that $\cap \mathcal{K}(t)$ contains the ball $B(0, r)$ with

$$
r=\sigma_{y}(1-2 \Delta) \frac{\sqrt{6}}{2} .
$$

The stress state $(0,0,0)$ thus satisfies the condition $(i)$ in Theorem 1 , with a value of $r$ given by expression (61). In view of applying Theorem 1 , we now turn our attention to condition (ii). This requires to determine the linear operator $\mathcal{L}(t)$ defined by (13). The matrix representation of $\mathcal{L}(t)$ in the orthonormal basis $\left(\boldsymbol{e}_{1}, \boldsymbol{e}_{2}\right)$ is found to be

$$
\mathcal{L}(t)=\left(\begin{array}{cc}
g_{1}(t) & 0 \\
0 & \frac{g_{1}(t)+2 g_{2}(t)}{3}
\end{array}\right) .
$$


We have

$$
\left.\|\mathcal{L}(t)\|=\max (\operatorname{eig} \mathcal{L}(t))=\max \left(g_{1}(t),\left(g_{1}(t)+2 g_{2}(t)\right) / 3\right)\right) .
$$

The constant $C$ in (44) can thus be taken as

$$
\left.C=\max \left(\sup \left(g_{1}\right), \sup \left(g_{1}+2 g_{2}\right) / 3\right)\right) .
$$

The value $\gamma(0, T)$ defined in (49) needs to be calculated. Using the expression (54) of $\mu(t)$, we find

$$
\mu(t)=g_{1}(t) \frac{1+2 h(t)}{2(2+h(t))}
$$

and

$$
\gamma(0, T)=\int_{0}^{T} \frac{3|\dot{h}(t)|}{2(h(t)+2)^{2}} d t
$$

where $h(t)=g_{2}(t) / g_{1}(t)=E_{1}(t) / E_{2}(t)$ is the ratio of the time-dependent Young's moduli in bars 1 and 2.

To be specific, take

$$
g_{1}(t)=\frac{1}{E}, g_{2}(t)=\frac{1}{E}\left(1+\delta \sin ^{2}\left(\pi \frac{t}{T}\right)\right)
$$

where $\delta$ and $E$ are fixed positive parameters. Calculating the integral in (62) gives $\gamma(0, T)=$ $E \delta /(3+\delta)$. Condition $(i)$ in Theorem 1 is satisfied with $\boldsymbol{\rho}^{*}=(0,0,0)$ provided that $\Delta \leq 1 / 2$, i.e.

$$
\frac{\sigma^{\theta}}{\sigma_{y}} \leq \frac{3}{2}
$$

Condition (ii) in Theorem 1 is found to be satisfied provided that

$$
9(p(\delta)-q(\delta))+3(3 p(\delta)+4 q(\delta)) \frac{\sigma^{\theta}}{\sigma_{y}}+(3 p(\delta)-4 q(\delta))\left(\frac{\sigma^{\theta}}{\sigma_{y}}\right)^{2} \leq 0
$$

where $p(\delta)=2 \delta^{2}(3+2 \delta)^{2}$ and $q(\delta)=\frac{3}{8}(3+\delta)^{2}$.

In Fig. 6 are represented the values $\left(\sigma^{\theta}, \delta\right)$ satisfying (64-65). For $\delta=0$, we obtain that shakedown occurs provided that $\sigma^{\theta} \leq 3 / 2 \sigma_{y}$, in agreement with the classical Melan's theorem for time-independent moduli. As can be observed on Fig. 6, the time fluctuations of the elastic moduli result in a decrease of the shakedown limit on $\sigma^{\theta}$. The shakedown limit vanishes for $\delta \simeq 0.38$, which corresponds to a time fluctuation of the Young's modulus of approximatively $27 \%$. Such a level of fluctuation is to be considered as unusually large: for ferrous alloys, the variation of the Young's modulus with temperature is about $1 \%$ per $100^{\circ} \mathrm{C}$, and variations of the temperature rarely exceed $200{ }^{\circ} \mathrm{C}$ in most applications. This corresponds to a fluctuation of the Young's modulus of $2 \%$. For such a level of fluctuation, the shakedown limit given by (64-65) is approximatively equal to $1.38 \sigma_{y}$, which corresponds to a drop of $8 \%$ compared to the case of time-independent elastic moduli.

Let us now compare the result (64-65) with the predictions of Theorem 2. The prescription (56) gives $\mu(t)=2 g_{1}(t)+g_{2}(t)$. Noting that the Young's moduli satisfy (28) with $\alpha=1 / E$ and $\beta=(1+\delta) / E$, condition (ii) in Theorem 2 is found to give

$$
3(4 s(\delta)-3)+12(s(\delta)+1) \frac{\sigma^{\theta}}{\sigma_{y}}+4(s(\delta)-1)\left(\frac{\sigma^{\theta}}{\sigma_{y}}\right)^{2} \leq 0
$$




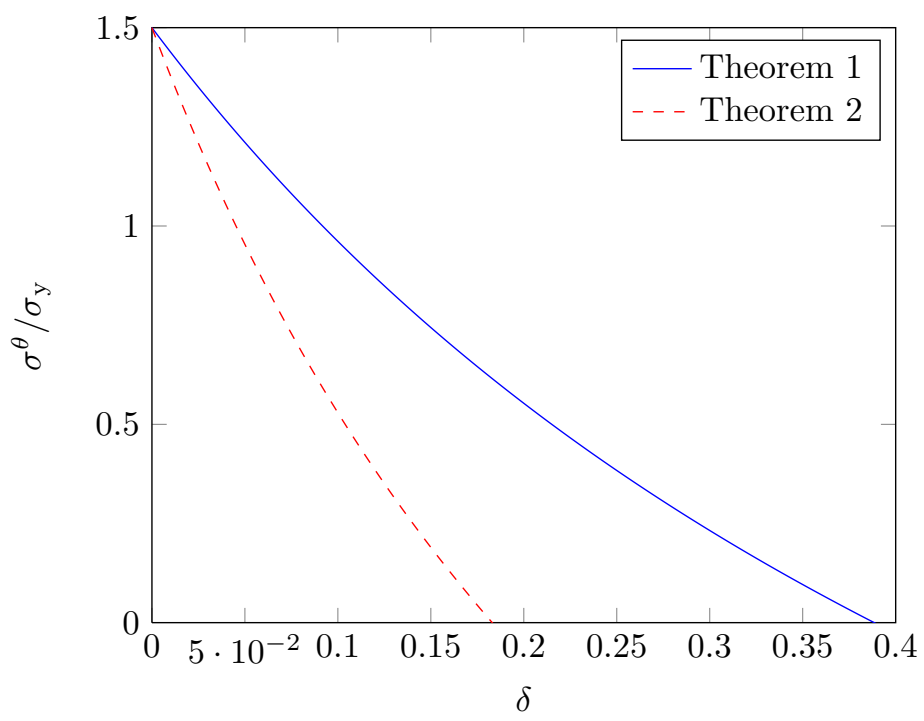

Figure 6: Shakedown limit in the case $F=0$.

with $s(\delta)=16 \delta^{2}(\delta+1)^{2}$. The values $\left(\delta, \sigma^{\theta}\right)$ satisfying (66) are bounded by the red dashed curve in Fig.6. As can observed in Fig. 6, Theorem 2 leads to pessimistic predictions regarding the shakedown limits compared to Theorem 1. Moreover, the gap between the results of Theorem 1 and Theorem 2 increases with the parameter $\delta$, i.e. with the time-fluctuations of the Young's moduli.

\subsection{Shakedown domains in the space of load parameters}

We now consider the general case $\sigma^{F} \neq 0$. In a similar way to $(7.2)$, the set $\mathcal{K}(t)$ can be written as

$$
\mathcal{K}(t)=\sqrt{6} \frac{\epsilon^{\theta}(t)}{g_{1}(t)+2 g_{2}(t)} \boldsymbol{e}_{2}+\mathcal{K}^{\prime}
$$

where $\mathcal{K}^{\prime}$ is a time-independent convex subset of $\mathcal{H}$. The exact expression of $\mathcal{K}^{\prime}$ depends on $\sigma^{F}$ and is not reported here, for the sake of conciseness. We simply mention that the set $\mathcal{K}^{\prime}$ is found to have a hexagonal shape if $\left|\sigma^{F}\right|<\sigma_{Y}$, and a triangular shape if $\left|\sigma^{F}\right| \geq \sigma_{Y}$.

For given time-dependence $g_{1}(t)$ and $g_{2}(t)$ of the Young's moduli, we aim at determining the values $\left(\sigma^{F}, \sigma^{\theta}\right)$ satisfying Theorem 1 . The expressions obtained previously for $C, \mu(t)$ and $\gamma(0, T)$ remain unchanged. The main difference with the case $\sigma^{F}=0$ is that the stress state $(0,0,0)$ is not necessarily in $\mathcal{K}(t)$ and thus cannot be used as an elastic solution in condition $(i)$. A simple numerical procedure has been implemented to determine an elastic solution that fulfills condition (i) for given values of $\sigma^{F}$ and $\sigma^{\theta}$. It consists in finding the largest incircle of the convex set

$$
\bigcap_{\left|\epsilon^{\theta}\right| \leq \sigma^{\theta} / E} \bigcap_{0 \leq t \leq T} \mathcal{L}(t)\left(\sqrt{6} \frac{\epsilon^{\theta}(t)}{g_{1}(t)+2 g_{2}(t)} e_{2}+\mathcal{K}^{\prime}\right) .
$$

If $\boldsymbol{\eta}$ and $r^{*}$ denote the center and radius of that circle, it can easily be seen that $\boldsymbol{\rho}^{*}(t)=\mathcal{M}(t) \boldsymbol{\eta}$ in an elastic solution, and that $B\left(\boldsymbol{\rho}^{*}(t), r^{*} / C\right) \subset \mathcal{K}(t)$ for all $t$. In practice, the time interval $[0, T]$ is 


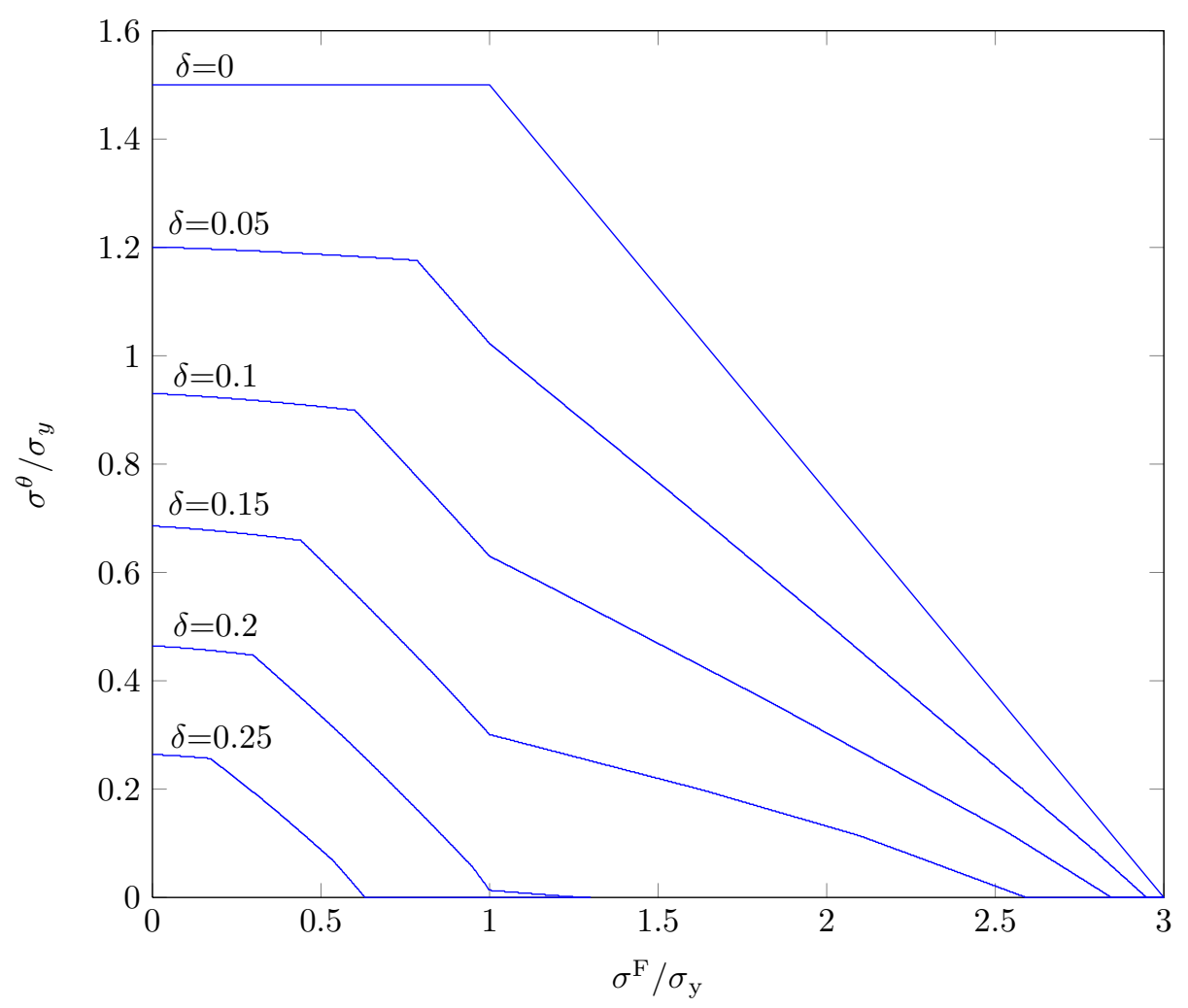

Figure 7: Shakedown domains for different levels of fluctuation of the elastic moduli.

discretized in a finite number of steps $t_{1}, \cdots, t_{n}$. Similarly, the interval $\left[-\sigma^{\theta}, \sigma^{\theta}\right] / E$ is discretized in a finite number of values $\epsilon_{1}^{\theta}, \cdots, \epsilon_{m}^{\theta}$. Such a discretization allows the convex set in (67) to be approximated by the convex polygon

$$
\bigcap_{1 \leq j \leq m} \bigcap_{1 \leq i \leq n} \mathcal{L}\left(t_{i}\right)\left(\sqrt{6} \frac{\epsilon_{j}^{\theta}}{g_{1}\left(t_{i}\right)+2 g_{2}\left(t_{i}\right)} \boldsymbol{e}_{2}+\mathcal{K}^{\prime}\right) .
$$

Finding the largest incircle of that polygon is a linear programming problem than can be solved using an interior point method (Orlowski and Pachter, 1987).

The shakedown domains delivered by that procedure are represented in Fig. 7 for different values of $\delta$. The functions $g_{1}$ and $g_{2}$ are taken in the form (63). The domain obtained for $\delta=0$ corresponds to Eq. (58). For some values of $\delta$, the shakedown domain is not convex, in agreement with the observations of Hasbroucq et al. (2010). As usual in shakedown analysis, knowing the complete time history of the loading $\epsilon^{\theta}(t)$ is not needed: the results in Fig. 7 only depend on the function $\epsilon^{\theta}(t)$ through the extreme value $\sigma^{\theta}=E \sup \left|\epsilon^{\theta}(t)\right|$.

\subsection{Incremental analysis}

In this section we present some numerical solutions of Eq. (15) obtained for specific functions $E_{1}(t), E_{2}(t), \epsilon^{\theta}(t)$ verifying Theorem 1. The curves in Fig.8(left) show the evolution of the residual 

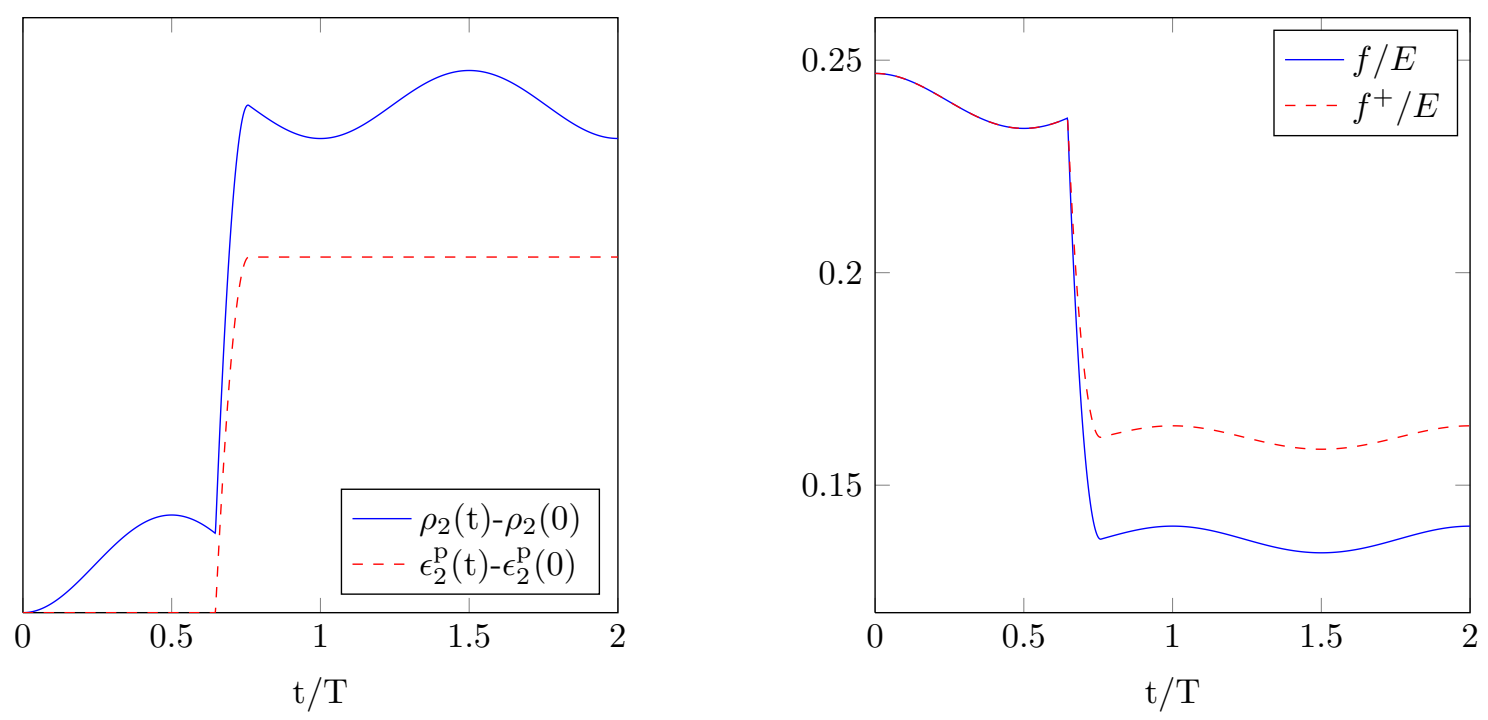

Figure 8: Residual stress and plastic strain in bar 2 (left); bound $f^{+}$on the elastic energy $f$ (right).

stress and of the plastic strain in bar 2 in the case $E_{1}(t)=E, E_{2}(t)=E /\left(1+0.1 \sin ^{2}(\pi t / T)\right)$, $\epsilon^{\theta}(t)=0.1 \sin (2 \pi t / T), F=0$. The initial state $\rho(0)$ is $(0.46,-0.53,0.07) E$. As expected from Theorem 1 , the residual stress converges towards a periodic solution and the plastic strain converges towards a stabilized value. In Fig.8(right) is presented an illustration of the energy bound (50) that plays an important role in this paper. The solid line in Fig. 8(right) shows the time evolution of the elastic energy $f(t)=\langle\boldsymbol{\rho}(t), \mathcal{L}(t) \boldsymbol{\rho}(t)\rangle / 2$, using the same data as in Fig 8(left). The dotted curve in Fig. 8(right) is the upper bound $f^{+}$on $f$ that is deduced from (50) using $\mu(t)=1$, that is

$$
f^{+}(t)=\frac{1}{2}\langle\boldsymbol{\rho}(0), \mathcal{L}(0) \mathcal{M}(t) \mathcal{L}(0) \boldsymbol{\rho}(0)\rangle+(3 C M \gamma(0, T)-r) \int_{0}^{t}\|\boldsymbol{\eta}(s)\| d s .
$$

As can be observed in Fig. 8, the upper bound $f^{+}$captures some of the non monotonic variations of $f$.

In Fig. 9 are plotted some trajectories of the residual stress in the space $\mathcal{H}$, as obtained for different initial states. The functions $g_{1}$ and $g_{2}$ are taken as $g_{1}(t)=\left(1+0.1 \cos ^{2}(\pi t / T)\right) / E$, $g_{2}(t)=\left(1+0.1 \sin ^{2}(\pi(t / T-1 / 4))\right) / E$. The closed loops in Fig. 9 correspond to elastic solutions. After possibly some complex transient path, all trajectories converge towards $T$-periodic elastic solutions, which is the main conclusion of Theorems 1-2. Notice the symmetry of the trajectories with respect to the axis $x_{1}=0$. Comparing two symmetric trajectories makes it clear that the distance between two solutions does not decrease with time. The plots in Fig. 9 are to be contrasted with the case of time-independent elastic moduli $g_{1}=g_{2}=1 / E$, represented in Fig. 10, for which the stress converges towards a stationary state and the distance between two solutions is decreasing with time. 


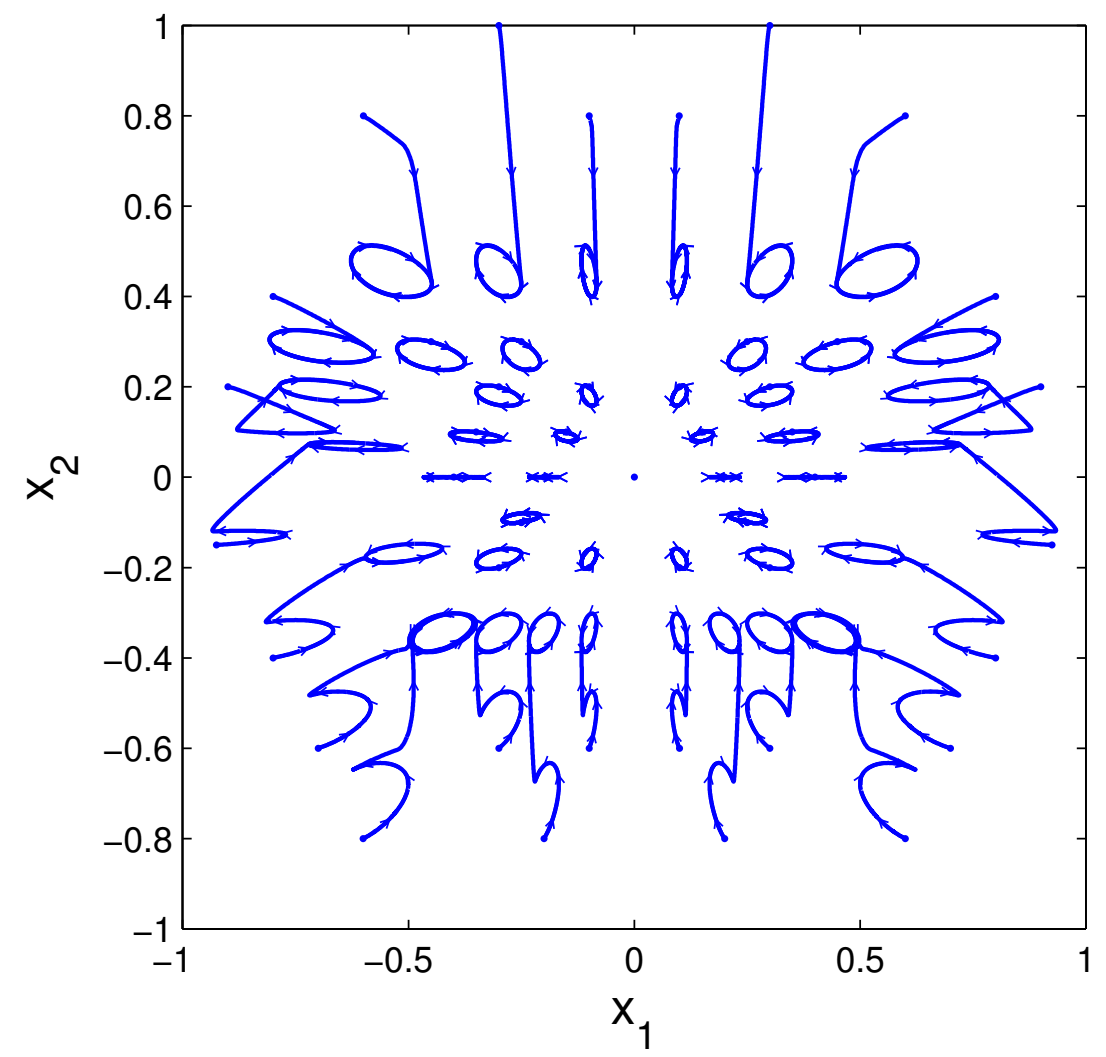

Figure 9: Trajectories of the stress state, case of time-dependent elastic moduli.

\section{Concluding remarks}

In this paper we have studied the asymptotic behavior of elastic-perfectly plastic media with temperature-dependent (or more generally time-dependent) elastic moduli. The shakedown theorems presented in this paper give sufficient conditions for the stress field to converge towards an elastic solution, independently on the initial state. The statement and the proof of the presented theorems differ significantly from the case of time-independent elastic moduli. The contraction property of the sweeping process is one of the main ingredient used to prove shakedown theorems in the classical case. Although that property does not longer hold when the elastic moduli vary in time, carefully studying the variations of the elastic energy still provides a key to solve the problem, as we have seen. An important result is that the time-variations of the elastic moduli should not be too large (in the sense of condition (ii) in Theorems 1-2) for shakedown to occur in a pathindependent fashion. As a result, there is a trade-off between the mechanical loading parameters and the time fluctuations of the elastic moduli: the larger the time fluctuations are, the smaller the load carrying capacity is (with respect to shakedown). A lot of progress remains to be made in the study of plasticity with time-dependent properties, and we only list a few open questions. First, 


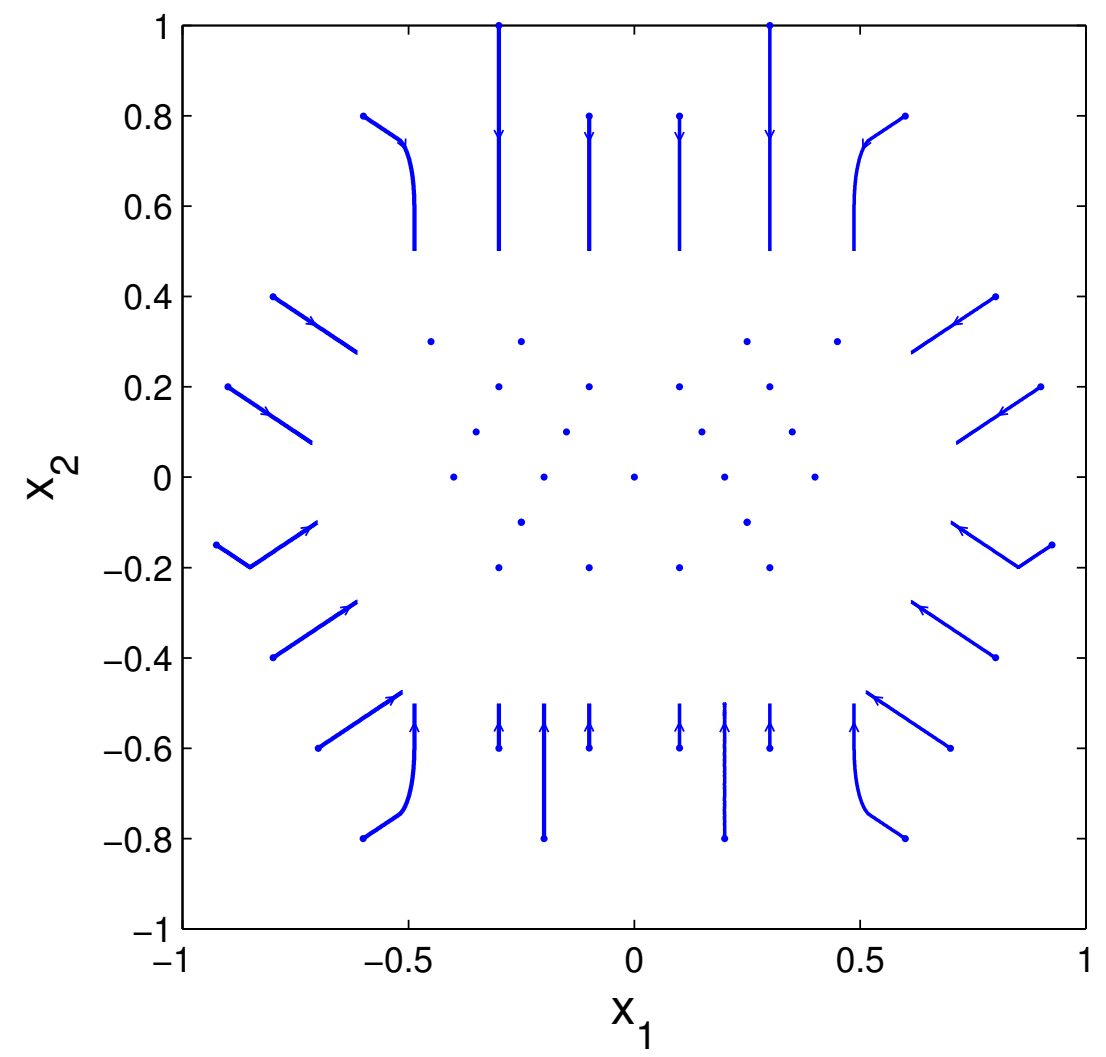

Figure 10: Trajectories of the stress state, case of time-independent elastic moduli.

it is possible that the conditions $(i)-(i i)$ in the proposed theorems could be partially relaxed, especially for infinite-dimensional systems. The cases of hardening plasticity and viscoplasticity are of interest and should be studied. Finally, for cyclic loadings, the convergence of the stress field towards a cyclic response is not proved in the general case, notably when the set of elastic solutions is empty. The ideas and techniques presented in this paper could possibly be useful to tackle such questions.

\section{A. An alternate proof for radial variations of the elastic moduli}

In this appendix, the linear operator $\mathcal{M}(t)$ is assumed to be of the radial form (53), so that $\mathcal{L}(t)=\mathcal{L}_{0} / \lambda(t)$ where $\mathcal{L}_{0}$ is time-independent. In that special case, a change of variables can be used to prove that the condition $(i)$ in Theorem 1 is sufficient to ensure shakedown.

Setting $\tilde{\boldsymbol{\rho}}(t)=\boldsymbol{\rho}(t) / \lambda(t)$, Eq. (15) becomes

$$
-\mathcal{L}_{0} \frac{d \tilde{\boldsymbol{\rho}}}{d t} \in \partial I_{\mathcal{K}_{(t)}}(\lambda(t) \tilde{\boldsymbol{\rho}}(t))
$$


Consider the convex set $\tilde{\mathcal{K}}(t)$ defined as $\tilde{\mathcal{K}}(t)=\mathcal{K}(t) / \lambda(t)$. It can easily verified from the general definition (4) of the subgradient that

$$
\partial I_{\tilde{\mathcal{K}}_{(t)}}(\tilde{\boldsymbol{\rho}})=\partial I_{\mathcal{K}_{(t)}}(\lambda(t) \tilde{\boldsymbol{\rho}})
$$

Eq. (68) can thus be rewritten as

$$
-\mathcal{L}_{0} \frac{d \tilde{\boldsymbol{\rho}}}{d t} \in \partial I_{\tilde{\mathcal{K}}_{(t)}}(\tilde{\boldsymbol{\rho}}(t)) .
$$

That last equation as the same structure as the differential inclusion (18) corresponding to time-independent elastic moduli.

Let $\boldsymbol{\rho}^{*}$ be an elastic solution for (15) (on a time interval $[\tau,+\infty[$ ), satisfying condition $(i)$ for some $r>0$. We note that $\tilde{\boldsymbol{\rho}}^{*}=\boldsymbol{\rho}^{*} / \lambda(t)$ is time-independent and belongs to $\tilde{\mathcal{K}}(t)$ for all $t \geq \tau$. Let $\boldsymbol{\rho}^{\prime} \in B\left(\boldsymbol{\rho}^{*}(t), r\right)$. Setting $\tilde{r}=r / \sup \lambda$, we now show that $B\left(\tilde{\boldsymbol{\rho}}^{*}, \tilde{r}\right) \subset \tilde{\mathcal{K}}(t)$. Consider a given $\tilde{\boldsymbol{\rho}}^{\prime} \in B\left(\tilde{\boldsymbol{\rho}}^{*}, \tilde{r}\right)$ and set $\boldsymbol{\rho}^{\prime}=\lambda(t) \tilde{\boldsymbol{\rho}}^{\prime}$. We have

$$
\left\|\boldsymbol{\rho}^{\prime}-\boldsymbol{\rho}^{*}\right\| \leq \lambda(t) \tilde{r} \leq r .
$$

Since $B\left(\boldsymbol{\rho}^{*}(t), r\right) \subset \mathcal{K}(t)$, we obtain $\tilde{\boldsymbol{\rho}}^{\prime} \in \tilde{\mathcal{K}}(t)$. We have therefore $B\left(\tilde{\boldsymbol{\rho}}^{*}, \tilde{r}\right) \subset \tilde{\mathcal{K}}(t)$, so that $\tilde{\boldsymbol{\rho}}^{*}$ satisfies Condition 1 for the differential inclusion (68). It follows from the results of Sec. 3 that all the solutions of (68) converge towards a time-independent state, i.e. that all the solutions of (15) converge towards a solution of the form $\boldsymbol{M}(t) \boldsymbol{\eta}$ (where $\boldsymbol{\eta}$ is time-independent).

\section{References}

E. Melan, Theorie statisch unbestimmter Systeme aus ideal-plastischen Baustoff, Sitz.Berl.Ak.Wiss. 145 (1936) 195-218.

P. S. Symonds, Shakedown in continuous media, J. Appl. Mech. 18 (1951) 85-89.

W. T. Koiter, General problems for elastic solids, Progress in solid mechanics, 1960.

K. DangVan, I. V. Papadopoulos, Introduction to fatigue analysis in mechanical design by the multiscale approach, Springer, 57-88, 1999.

D. C. Pham, On shakedown theory for elastic-plastic materials and extensions, J. Mech. Phys. Solids 56 (2008) 1905-1915.

Q. S. Nguyen, On shakedown analysis in hardening plasticity, J. Mech. Phys. Solids 51 (2003) $101-125$.

A. Corigliano, G. Maier, S. Pycko, Dynamic shakedown analysis and bounds for elastoplastic structures with nonassociative, internal variable constitutive laws, Int. J. Solids. Struct. 32 (21) (1995) 3145-3166.

G. Bodovillé, G. De Saxcé, Plasticity with non-linear kinematic hardening: modelling and shakedown analysis by the bipotential approach, Eur. J. Mech. A 20 (1) (2001) 99-112.

Y. J. Ahn, E. Bertocchi, J. Barber, Shakedown of coupled two-dimensional discrete frictional systems, J. Mech. Phys. Solids 56 (12) (2008) 3433-3440. 
M. Peigney, Shakedown theorems and asymptotic behaviour of solids in non-smooth mechanics, Eur. J. Mech. A 29 (5) (2010) 784-793.

J. Zarka, J. Frelat, G. Inglebert, A New Approach to Inelastic Analysis of Structures, Martinus Nijhoff Publishers, Dordrecht, 1988.

H. Maitournam, B. Pommier, J. Thomas, Détermination de la réponse asymptotique d'une structure anélastique sous chargement cyclique, C.R. Mecanique 330 (2002) 703-708.

V. Carvelli, Z. Cen, Y. Liu, G. Maier, Shakedown analysis of defective pressure vessels by a kinematic approach, Archive of Applied Mechanics 69 (9-10) (1999) 751-764.

M. Peigney, C. Stolz, Approche par contrôle optimal des structures élastoviscoplastiques sous chargement cyclique, C.R.Acad.Sci. Paris, II 329 (2001) 643-648.

M. Peigney, C. Stolz, An optimal control approach to the analysis of inelastic structures under cyclic loading, J. Mech. Phys. Solids 51 (2003) 575-605.

J.-W. Simon, D. Weichert, Shakedown analysis of engineering structures with limited kinematical hardening, International Journal of Solids and Structures 49 (15) (2012) 2177-2186.

K. V. Spiliopoulos, K. D. Panagiotou, A direct method to predict cyclic steady states of elastoplastic structures, Computer Methods in Applied Mechanics and Engineering 223 (2012) 186-198.

D. Weichert, A. Ponter, A Historical View on Shakedown Theory, in: E. Stein (Ed.), The History of Theoretical, Material and Computational Mechanics-Mathematics Meets Mechanics and Engineering, Springer, 169-193, 2014.

G. Borino, Consistent shakedown theorems for materials with temperature dependent yield functions, Int. J. Solids. Struct. 37 (22) (2000) 3121-3147.

J. König, A shakedown theorem for temperature dependent elastic moduli, Bull. Ac. Pol. Sci., Ser. Sci. Techn. 17 (3) (1969) 161-165.

B. Halphen, S. di Domizio, Evolution des structures élastoplastiques dont les coefficients d'élasticité dépendent de la température, 17ème congrès français de mécanique, Troyes, 2005.

S. Hasbroucq, A. Oueslati, G. De Saxcé, Inelastic responses of a two-bar system with temperaturedependent elastic modulus under cyclic thermomechanical loadings, Int. J. Solids. Struct. 47 (14) (2010) 1924-1932.

S. Hasbroucq, A. Oueslati, G. De Saxcé, Analytical study of the asymptotic behavior of a thin plate with temperature-dependent elastic modulus under cyclic thermomechanical loadings, Int. J. Mech. Sci. 54 (1) (2012) 95-104.

J. J. Moreau, Evolution problem associated with a moving convex set in a Hilbert space, J. Diff. Eq. 26 (3) (1977) 347-374.

H. Brézis, Opérateurs maximaux monotones et semigroupes de contractions dans les espaces de Hilbert, North-Holland, Amsterdam, 1972.

R. T. Rockafellar, Convex analysis, vol. 28, Princeton University Press, 1997. 
R. Hill, The mathematical theory of plasticity, Oxford university press, 1950.

H. Lanchon, Elastic-plastic torsion of a cylindrical shaft with simply or multiply connected cross section, J. Mecanique 13 (1974) 267-320.

B. Halphen, Elastic perfectly plastic structures with temperature dependent elastic coefficients, C.R. Mécanique 333 (8) (2005) 617-621.

B. Halphen, Stress accommodation in elastic perfectly plastic and viscoplastic structures, Mech. Res. Comm. 2 (1976) 273-278.

J. Baillon, A. Haraux, Comportement à l'infini pour les équations d'évolution avec forcing périodique, Arch. Rational Mech. Anal. 67 (1) (1977) 101-109.

E. Wesfreid, Asymptotic behaviour of an elastoviscoplastic material submitted to a cyclic load, Proc. Roy. Soc. Edin. A 89 (3-4) (1981) 189-199.

P. Ballard, Frictional Contact Problems for Thin Elastic Structures and Weak Solutions of Sweeping Processes, Arch. Rational Mech. Anal. 198 (3) (2010) 789-833.

O. Débordes, B. Nayroles, et al., Sur la théorie et le calcul à l'adaptation des structures élastoplastiques., J. Mecanique 15 (1) (1976) 1-53.

W. Rudin, Real and complex analysis, McGraw-Hill, 1987.

L. Schwartz, Analyse mathématique, vol. 94, Hermann Paris, 1967.

B. Mercier, Sur la théorie et l'analyse numérique de problèmes de plasticité, Ph.D. thesis, Université Paris VI, 1977.

M. Orlowski, M. Pachter, Linear programming in $R^{3}$ and the skeleton and largest incircle of a convex polygon, Comp. Math. Appl. 13 (4) (1987) 401-405. 